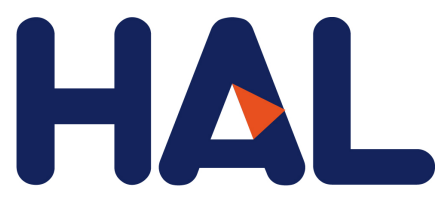

archives-ouvertes

\title{
Exegesis of Sect. II and III.A from " Fundamentals of the Mechanics of Continua " by E. Hellinger
}

\author{
Simon R. Eugster, Francesco Dell'Isola
}

\section{To cite this version:}

Simon R. Eugster, Francesco Dell'Isola. Exegesis of Sect. II and III.A from " Fundamentals of the Mechanics of Continua " by E. Hellinger. 2017. < hal-01551110>

\section{HAL Id: hal-01551110 \\ https://hal.archives-ouvertes.fr/hal-01551110}

Submitted on 30 Jun 2017

HAL is a multi-disciplinary open access archive for the deposit and dissemination of scientific research documents, whether they are published or not. The documents may come from teaching and research institutions in France or abroad, or from public or private research centers.
L'archive ouverte pluridisciplinaire HAL, est destinée au dépôt et à la diffusion de documents scientifiques de niveau recherche, publiés ou non, émanant des établissements d'enseignement et de recherche français ou étrangers, des laboratoires publics ou privés. 


\title{
Exegesis of Sect. II and III.A from "Fundamentals of the Mechanics of Continua"** by E. Hellinger
}

\author{
Simon R. Eugster ${ }^{1,2 * *}$ and Francesco dell'Isola ${ }^{3,2}$ \\ ${ }^{1}$ Institute for Nonlinear Mechanics, University of Stuttgart, Stuttgart, Germany \\ 2 International Research Center for the Mathematics and Mechanics of Complex Systems, MEMOCS, Università \\ dell' Aquila, L'Aquila, Italy \\ ${ }^{3}$ Dipartimento di Ingegneria Strutturale e Geotecnica, Università di Roma La Sapienza, Rome, Italy
}

Received XXXX, revised XXXX, accepted XXXX

Published online XXXX

Key words Ernst Hellinger, virtual work, continuum mechanics, foundations of kinetics, constitutive laws

This is a second exegetic essay on the fundamental review article DIE ALLGEMEINEN ANSÄTZE DER MECHANIK DER KONTINUA in the Encyklopädie der mathematischen Wissenschaften mit Einschluss ihrer Anwendungen, Bd. IV-4, Hft. 5 (1913) by Ernst Hellinger which concerns the translation and the commentary of pp. 629-662. Within these pages, the setting of the basic constitutive equations for field theories, whose formulations are based on the principle of virtual work or on the principle of stationary action, are discussed. The interest for a contemporary reader for the herein presented subject is still substantial, as this article clearly contains some considerations and visions being still topical. However, there is also an epistemological interest in examining it from the point of view of a historian of science. Indeed, it represents an available but forgotten source of an important piece of mechanical sciences. Available, because it is still present in our libraries in its complete form, but forgotten because, being written in German by a Jewish refugee escaped to the United States, has been ignored by the main stream of the dominant groups in continuum mechanics. The ideas by Hellinger and the German school of continuum mechanics (remark that this school includes, as we have discovered reading this article, even Gauss himself!) were lost or dramatically deformed in translation. We believe that the destiny of Hellinger's paper is an evidence supporting Lucio Russo's view about history of science. Our aim is to trace the origins of current ideas of mechanical sciences to their original sources.

Copyright line will be provided by the publisher

\section{Introduction}

As in the previous paper [23] of this exegetic series, the presented English translation of the original text written in German by Hellinger is typed in italic and is indented on both sides. The comments and explanatory remarks are in standard text style. We tried to produce a word-by-word translation totally refraining from allowing us to include any comment or interpretation of the original text because of translation. When we were obliged to introduce a word which had no correspondence in the German text, we included it in square brackets as follows: [xxxx]. For more general considerations about Hellinger's masterpiece, we refer to the introduction of [23]. Additionally, we remark that some translated excerpts of Hellinger's paper can also be found in Maugin [46]. In the present introduction we want to develop some considerations which may be of utility not only to frame Hellinger's contribution to our understanding of continuum mechanics, but also to examine other contexts, in particular those discussions where the attribution of a text to an author is questioned, denied or, on the contrary, strongly supported.

For the moment, we propose the following imaginary situation to the reader: let us assume that the article by Hellinger, which we are translating here, in a far future (but if human beings will not be careful such a future may not be so far, unfortunately) may be so corrupted in its transmission that the author's name is lost. It could even happen that then a courageous, but a little bit naive, philologist and historian of science arrives to have a conjecture about its authorship. The imaginary future philologist and historian, which we will call the Courageous Philologist, may arrive at the following chain of ideas: the article which he holds in his hands is of very high quality (this he manages to understand) and it deals with continuum mechanics. He can more or less determine the presumed period when this article has been written and he discovers that it was indeed written in a period very close to the age in which two famous experts of continuum mechanics

* DIE ALLGEMEINEN ANSÄTZE DER MECHANIK DER KONTINUA. Encyklopädie der mathematischen Wissenschaften mit Einschluss ihrer Anwendungen. Bd. IV-4, Hft. 5 (1913).

** Corresponding author E-mail: eugster@inm.uni-stuttgart.de, Phone: +4971168568152 
flourished. He therefore conjecturally attributes the article to Truesdell and Noll. As a further strong hint for this attribution he comes to know that Noll was of German origin and he could also find papers by Truesdell written in German (see e.g. [1]). Future philology and history of science may therefore be traversed by a violent, sharp and endless dispute about the question of authenticity of the article entitled DIE ALLGEMEINEN ANSÄTZE DER MECHANIK DER KONTINUA (henceforth denoted by the acronym DAADMDK). This kind of disputes is not rare. To give an example, the reader is

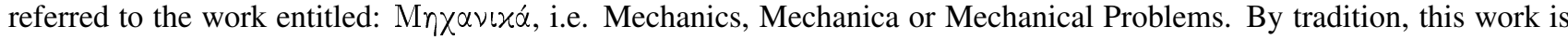
attributed to Aristotle but its authorship has been questioned also in ancient sources (cf. the Loeb edition of Aristotle's Minor Works [3]). While Winter [91] suggests with really persuading arguments that the author was Archytas of Tarentum, Coxhead [11] claims that it is only possible to conclude that the author was one of the Peripatetics. What is certain is only that during the Renaissance an edition of this work was published by Francesco Maurolico. Many future historians of mechanics in the imagined dispute, henceforth called the Skeptical Scholars, will attack the Courageous Philologist by stating that:

i) The style of the DAADMDK is too inhomogeneous and it is thus not reasonable to assume that it is authored by a single author. Indeed, they will observe that at the beginning of No. 2 (see [23]) a very primitive notation for placements is introduced recalling the one used in various works written between 1824 and 1850 by an author using Italian language which is also cited in Truesdell and Toupin [82]. Most likely the author's name is Gabrio Piola, see [18], whose traces would be lost if not two kinds of stress tensors were named after him: it is anyway disputable that Piola is so primitive, as his name is used very often together with the name of Kirchhoff. The Skeptical Scholars will add that the evidence of multi-authorship is then given by footnote 8) on page 607 of DAADMDK (see also footnote 19 in [23]) which is a clear missing link between pre-Levi-Civita tensorial notation and mature Levi-Civita one. They will remark that this intermediate notation towards a Levi-Civita one is sometimes abandoned in the text and then resumed. They will conclude stating that formula (12a) appearing in No. 16 of DAADMDK (to be translated in the forthcoming last paper of this series) is formulated with a mature Levi-Civita notation and that all these discrepancies definitively prove that the hands who wrote different parts of DAADMDK are not the same.

ii) The authors of DAADMDK must be aware of the contributions by Noll, therefore at least one of them must be posterior to Noll. Indeed, the Skeptical Scholars will observe that part III-A of DAADMDK, which includes Nos. 6, 7 and 8, makes extensive use of the principle of objectivity ${ }^{1}$ unanimously attributed to Noll in the literature which reached them. In their opinion, the apparent anachronism can be solved only by assuming that non-contemporary authors in different times contributed to DAADMDK. Moreover, the greatest lack of internal coherence in the presented matter becomes obvious in No. 4 of Part I of DAADMDK. The much later theories, called generalized continuum theories or theories of structured continua appear in this number. These theories were clearly unknown to Truesdell and Noll, which together with their followers, believe that they are not logically consistent.

iii) The text of DAADMDK is clearly corrupted. In any case, it cannot be attributed to Truesdell and Noll as it is based on the principle of virtual displacements, which has been understood and developed later (see e.g. [35] and [26]). About this point, the Skeptical Scholars do not have unanimous opinions. Indeed, some of them remark that Truesdell and Toupin introduce in a previous work some variational principles in continuum mechanics. This undoubted and undisputed philological observation is explained in two ways: a) by assuming that also Truesdell and Toupin is a corrupted text, where some subsequent authors or revisers added some references to variational principles or b) by assuming that Toupin is a later reviser of Truesdell's works who was aware of the later development of continuum mechanics, eventually based on the principle of virtual work. Another controversy among the Skeptical Scholars concerns the relationship between the principle of virtual displacements and the principle of virtual work. Some among them claim that the second one is the more advanced version of the first one, even if they cannot establish in what exactly this last improvement consists. Others claim the principles to be the same but named differently.

iv) The text of DAADMDK shows a mathematical competence which seems much more advanced than the mechanical competence. The authors which wrote the mathematical parts must be of a later period than the authors of the mechanical parts. Here the Skeptical Scholars are unanimous. It is clear that in DAADMDK the understanding of the concept of Fréchet and Gâteaux derivatives is rather deep and sophisticated. Furthermore the Lemma of Urysohn (at least in its form valid in the three-dimensional Euclidean space endowed with natural topology) is mastered with clever applications and the method of Lagrange multipliers is used in its full potentiality.

1 To this (and the same) principle many different names were given. Piola did not formulate explicit axioms for naming the assumption expressed by this principle, but formulates them clearly in formulas. In particular, see the considerations which led Piola to write Eq. (10) on p. 60 in No. 35 of Chap. I "Memoria intorno alle equazioni fondamentali del movimento di corpi qualsivogliono considerati secondo la naturale loro forma e costituzione" of [18], cf. also Eq. (12) in [14]. In [80], Truesdell seems to recover the spirit of Piola by calling it either principle of material frame-indifference or invariance under superposed rigid motions. 
v) Both the Skeptical Scholars and the Courageous Philologist are puzzled by the historical references to Lagrange (an author openly despised by Truesdell), to Hamilton and to the Cosserat brothers. Concerning this point they all do agree that further investigations are required. They are also puzzled by the reference to relativity. This point seems to cause them serious troubles and they tend to assume that this part was added by a much later reviser.

The reader should not believe that the previously described imaginary situation has absolutely no chances to become

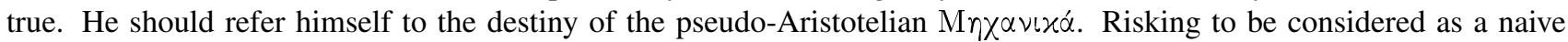
version of the Courageous Philologist, we dare to give our opinion about the destiny of such a famous text (see also McLaughlin [47]). The pseudo-Aristotelian M $\eta \chi \alpha \nu \iota x \alpha$ attracted our attention because it contains undoubtedly one of the first evidences of the application of the principle of stationarity of potential energy in equilibrium. The reader should remark that (if our sources are correct) Aristotelian dynamics postulates that in the absence of external interactions a body remains at rest. Therefore, the principle of inertia was most likely unknown to Aristotle. Russo [68], pp. 286-293, claims that Hipparchus of Nicaea was probably one of the first mechanicians to formulate a correct principle of inertia ${ }^{2}$. The fact that Aristotle had not yet in his hands the correct formulation of dynamics does not exclude that he could have had a correct formulation of statics. The attentive reader ${ }^{3}$ will agree that the passage from statics to dynamics is not easy and not immediate, especially if one has not in his hands the texts by D'Alembert, Lagrange or Hamilton (and also in this case one may have some troubles!). Accordingly it is possible that Aristotle could have correctly formulated the problem of the equilibrium of the lever. Hence, it cannot be excluded that the particular application of the principle of virtual work found in the pseudo-Aristotelian M $\rceil \chi \alpha \nu\llcorner x \alpha$ could have been written by Aristotle. In contrast, Winter [91] believes that the author of the complete M $\eta \chi \alpha \nu \iota x \alpha$ is Archytas. The attribution to Archytas of Tarentum of those parts involving the principle of virtual work is really appealing to the senior author: being himself a philosopher from Magna Graecia, he cannot be unhappy of such a conjecture. A lot has been written about M $\eta \chi \alpha \nu \iota x \alpha$. We will simply dare to add some remarks. As in our opinion the book by Russo [68] clearly places the birth of science and scientific technology in the Hellenistic period, we tend to disagree about the attribution to Archytas, if it is true that he flourished at least one century earlier. Moreover, if one has studied physics using a modern textbook (cf. for instance [2,37,41]), one will be surprised with the analogies which

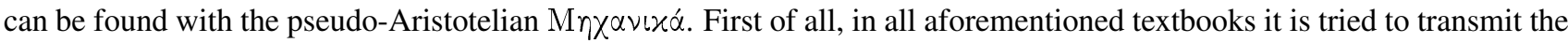
general ideas by means of "practical" examples of applications. Second, the general style tries to be friendly and to limit the use of mathematics to the minimum possible. Finally, they all provide a set of questions which can be supposedly asked during a student's exam together with a list of answers that a teacher expects to hear: all these textbooks seem to have been

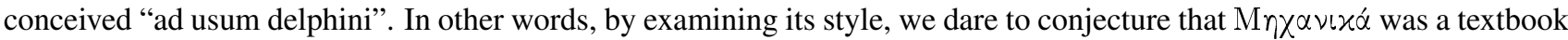
which was used to introduce the ancient students to mechanics and to its mathematical formulation exactly in the style of modern textbooks at university level. It seems to us very similar to an ancient version of the Berkeley physics course [41].

We conclude this introduction remarking that the style of Hellinger is different from the one adopted by the Berkeley physics course: Hellinger is a mathematical physicists. He shows the theory, its logical consistence and simply gives some hints to possible applications. The article by Hellinger is the intelligent compilation of many related topics and theories whose trait-d'union must be found in the fact that they are all formulated in terms of a field theory (as made explicit in Part III-A). The list of particular examples is given in Part III-B. Hellinger shows us how a (meta-)theory can be applied to supply the formulation of particular "concrete" theories. The reason for which Hellinger's article is, in our opinion, very original can be stated in other words as follows: Hellinger presents in an unified way all field theories which were already formulated at his times, assuming as fundamental paradigm for physics the concept of field (in contrast to the concept of particle). From this point of view Hellinger presents the modern point of a follower of Aristotle (see e. g. [90]). This does not imply that he did not believe in the discrete nature of matter (i.e. that he was not an Epicurean), but his article was intended to the aforementioned aim. It is remarkable that later epigones (see [81]) continued his work, however, never introducing any variational principle.

\section{Annotated translation of No. 5 (pp. 629-637)}

We start our commentaries by remarking that while evaluating the impact of a scientific text, its historical role and its overall relevance, it is very important to understand the exact nominalistic ${ }^{4}$ frame in which the author places his work.

\footnotetext{
2 This statement can be supported, for instance by remarking that Lucretius in "On the Nature of Things" [39], pp. 60-65, following most likely Epicurean School, stated that the "default state" of matter is motion, not stasis.

3 For an amusing definition of such a kind of reader go to http://www.ma.rhul.ac.uk/ uvah099/Sat/reader.html.

4 We use the word nominalism with the meaning given to it by Ockham (one of the founders of modern epistemology [76]). Indeed, exactly as done by his follower Lagrange, Ockham assumed the strongly argued position that while it is possible to state the existence of individuals, on the contrary the supra-individual universals (essences, or forms) have a secondary ontological status: universals are only the products of abstraction from individuals by the human mind and they have not any extra-mental existence.
} 
Many controversies raised among mechanicians which seem to have been caused by misunderstandings simply due to the different use of words. Moreover, many works seem to have as only true original content the change of names of wellknown abstract concepts, while they seem to be simply formulating very well-know statements concerning these concepts. Lagrange, in his masterpiece the "Mécanique Analytique" [42] not only frames his work in its historical context $\mathrm{t}^{5}$ but also starts immediately by clarifying its nominalistic choices. On page 1, on the second line of his treatise, he clearly specifies what he intends with the word force and he clearly specifies that for him this word is equivalent to the word puissance (i.e. power). This nominalistic choice is perfectly legitimate: however ${ }^{6}$, some detractors of his works (see Truesdell's Essays in the History of Mechanics [79]) have used a different nominalistic choice (their own!) for interpreting Lagrange. In this way they are lead to misleading conclusions and to misplace the contribution by Lagrange to mechanical sciences. On the contrary, Lagrange introduces on pp. 8-9 of [42] coherently with his lucid point of view the word moment in a non-conventional ${ }^{7}$ way. Indeed, at the end of p. 8 one can read (the upcoming English translations of Lagrange's excerpts in the footnotes are ours):

«Galilée entend par moment d'un poids ou d'une puissance appliquée à une machine, l'effort, l'action, l'énergie,

l'impetus de cette puissance pour mouvoir la machine [...] \& il fait voir que le moment est toujours proportionnel

à la puissance multipliée par la vitesse virtuelle, dépendante de la maniere dont la puissance agit.» ${ }^{8}$

It is therefore clear that Galileo and Lagrange with the word moment mean what nowadays is called (virtual) power. Consequently, it is not surprising that a distracted reader could completely misunderstand all the statements by Lagrange and believe that they are all non-sense. Naive Platonistic writers believe that their own nomenclature is intrinsically true and moreover that it is the only possible one. On p. 9 of [42], Lagrange states that Galilean nomenclature was adopted also by Wallis in his Mechanics published in 1670, i. e. [89]. Then Lagrange clarifies why in the common usage the word moment has assumed another (although related) technical meaning. On p. 9 one reads that:

«Aujourd'hui on n'entend plus communément pour moment, que le produit d'une puissance par la distance de sa direction à un point ou à une ligne, c'est-à-dire par le bras de levier par lequel elle agit; mais il me semble que la notion du moment donnée par Galilée \& par Wallis est bien plus naturelle \& plus générale, \& je ne vois pas pourquoi on l'a abandonnée pour y en substituer une autre qui exprime seulement la valeur du moment dans certains cas, comme dans le levier, \&c.»9

The reader should consider in detail the phenomenon of the shift in meaning which occurred to the word moment as it is documented without any doubt by the (easily available!) text by Lagrange. This phenomenon and its importance are fully described in [68]. Moment of a force (for Lagrange force is equivalent to power) is the energy expended per unit time by the force on a virtual velocity. Then in some cases (i.e. rigid virtual velocities) this moment can be calculated in terms of the force times its lever arm (multiplied by the virtual angular velocity!). Therefore Lagrange remarks that the general concept is confused with the particular one and that the particular concept manages to monopolize the word originally used in a more general sense. Note that Lagrange was visionary: he knew that the concept of moment expended by a force was important and regrets that only a particular application of this general concept appeared to be considered more important. It is ironic that Truesdellism gave such an importance to the word moment in its more particular meaning refusing to consider the importance of the (Galilean and Lagrangian) concept to which the word moment is referred in the more general case.

We conclude these preliminary considerations remarking that in the last decades the term dynamics has largely superseded kinetics in the textbooks of mathematical-physics and physics. The nomenclature used by Hellinger was rather common at the beginning of 20th century (one is refereed for instance to the very nice textbook of Wright [92]).

\section{The foundations of kinetics.}

5a. The equations of motion of the continuum. It is the task of kinetics to determine, what motion arises within the continuum considered so far, when force effects occur in it somehow specified in time, or vice versa,

\footnotetext{
5 Of course also Lagrange's opinions can be criticized: we cannot refrain from remarking that his vision of Hellenistic mechanics was at least reductive.

6 The careful discussion of this point will deserve more careful future discussion.

7 Of course with conventional we mean: following the conventions applicable in a given time, a given place and for a given group of persons.

8 Galileo means with moment of a weight or of a force (puissance) applied to a machine, the effort, the action, the energy, the impetus of this force for moving the machine [...] and he shows that the moment is always proportional to the force multiplied with the virtual velocity, depending on the way in which the force acts.

9 Nowadays one does mean more commonly for moment nothing else than the product of one force times the distance between its direction and one point or one line, that is [the force] times the lever arm through which it is acting; but it seems to me that the notion of moment given by Galileo and Wallis is much more natural and more general and I do not see why it has been abandoned to be replaced with another concept which expresses only the value of the moment in certain cases, as happens [when dealing] with the lever.
} 
which effects are required for the maintenance of a particular motion. As in statics, the effects are thereby thought as given by the coefficients of the expression of work $\delta A$, while the kind of their dependence on the function of motion remains open at first.

For now, we address only ordinary media considered in No. 3. The transition from statics to kinetics can occur as in the mechanics of discrete systems with the help of d'Alembert's Principle (cf. IV 1, No. 36, Voss); its generalization to continuous systems is offering itself almost automatically, if one let lead oneself as in statics (S. 616) by the idea of a limit process to the continuum, or if one proceeds directly in the sense of the analogy between point systems and continua. From these points of view, already Lagrange ${ }^{10}$ has tackled those problems of hydrodynamics which he considered.

Remark that already Hellinger is aware of the fact that the applications of the principle of virtual work to continuum mechanics dates back to the work of Lagrange himself. Accordingly, the first observation of this fact made by Piola (see [20]) is confirmed by the subsequent literature. Moreover, Hellinger already anticipates the possibility to deduce a continuum model from a discrete one by means of a limit process: of course we do not mean that he could perform this limit with the methods of Gamma-convergence.

According to [this last analogy] one can state the following principle which is in full correspondence with the notion of the general mechanics of continua developed by d'Alembert ${ }^{11}$ himself: Considering the forces and stresses which act during the motion at a particular instant of time on the quantum $V_{0}$ of the media, then they are in static equilibrium in the former sense, provided that one adds in every position additional forces ("forces of inertia"), whose components computed per unit of mass of the continuum are the same but opposite to the accelerations: ${ }^{12}$

$$
-\frac{\partial^{2} x}{\partial t^{2}}=-x^{\prime \prime},-\frac{\partial^{2} y}{\partial t^{2}}=-y^{\prime \prime},-\frac{\partial^{2} z}{\partial t^{2}}=-z^{\prime \prime}
$$

Even if it frequently seems to be convenient to put this principle as an axiom on the top of kinetics, the question remains, in which independent constituent parts one can divide it and how much are these independent of the static axioms - a question, which appears in the very same sense already in the mechanics of discrete systems. Therefore it should be noticed here just shortly, how such [a formulation of] d'Alembert's principle incorporates the statement being equivalent to Newton's second axiom: that the acceleration of an imagined freely moving volume element is equal to the sum of all applied forces to this element; that this principle incorporates on the other hand - which has firmly been pointed out by G. Hamel ${ }^{13}-$ a statement being logically independent of this first constituent part: If there are forces acting on a continuum, such that the accelerations of every particle following Newton's second law are admissible with respect to the systems kinematic constraints, then these accelerations really occur.

Before continuing to read Hellinger, it is worth to read directly what has been written by Lagrange. In [42], one reads at the end of p. 11, beginning of p. 12:

«Et en général je crois pouvoir avancer que tous les principes généraux qu'on pourrait peut-être encore découvrir dans la science de l'équilibre, ne seront que le même principe des vitesses virtuelles, envisagé différemment, \& dont ils ne différeront que dans l'expression.»14

By introducing now the principle of virtual displacements as equilibrium condition into d'Alembert's principle, one consequently obtains the variational principle used by Lagrange ${ }^{15}$ as basic formula in dynamics. For every instant $t$, one thinks of the motion as in No. 2, (6) superposed by an infinitesimal virtual displacement, which is admissible with respect to the kinematic constraints of the continuum arising at the instant of time $t$; then the virtual work augmented by the inertia forces must vanish always:

$$
-\iiint_{(V)} \varrho\left(x^{\prime \prime} \delta x+y^{\prime \prime} \delta y+z^{\prime \prime} \delta z\right) d V+\delta A=0
$$

10 Cf. in particular Méc. anal., 2. part., Sect. XI, §I.

11 Traité de dynamique, Paris 1743. Cf. IV 1 Voss, p. $77^{209}$ ).

12 In the following, apostrophes denote the derivatives of the functions of motion (1) with respect to $t$ for constant a, $b, c$.

13 G. Hamel, Math. Ann. 66 (1908), p. 354; p. 386 the independence for the mechanics of rigid bodies is demonstrated in an example; cf. also the extensive presentation in Hamel's Elementarer Mechanik, p. $306 f$.

14 And in general I believe to be able to conjecture that all the general principles which may be, possibly, discovered in the science of equilibrium will be nothing else than the principle of virtual velocities conceived in a different way and from which they will differ simply in their expression.

15 Méc. analyt., 2. part., Sect. II. 
and this for every instant of time t of the path of motion. For the case of an arbitrarily continuously deformable body and under the same assumptions as in No. $3 \mathrm{c}$, one can deduce as equations of the motion for every point of the continuum at any instant of time [the following ones]:

$$
\varrho x^{\prime \prime}=\varrho X+\frac{\partial X_{x}}{\partial x}+\frac{\partial X_{y}}{\partial y}+\frac{\partial X_{z}}{\partial z} \quad\left(\begin{array}{c}
x, y, z \\
X, Y, Z
\end{array}\right),
$$

while the boundary conditions (5b) of No. 3 remain unchanged for every instant of time $t$. However, these equations only determine the motion, when the connection of the force and the stress components with the functions of motion is specified.

Concerning the consideration of kinematic constraints, we refer here exclusively to the case of so called holonomic constraints, which contain no time derivatives of the functions of motion. ${ }^{16}$ Such a requirement differs from the form considered in No. $4 \mathrm{c}$ only by the explicit appearance of $t$ :

$$
\omega\left(a, b, c ; x, y, z ; x_{a}, \ldots, z_{c} ; t\right)=0 \text {. }
$$

Now, for the virtual displacements this form of condition at time $t$ comes into question only; the varied position shall (for arbitrarily small $\sigma$ ) satisfy the condition (3) for the considered fixed value of $t$, such that by differentiating with respect to $\sigma$ ("variation of the motion for fixed $t$ ") it follows

$$
\sum_{(x y z)} \frac{\partial \omega}{\partial x} \delta x+\sum_{(x y z, a b c)} \frac{\partial \omega}{\partial x_{a}} \delta x_{a}=0 \quad \text { for every } t
$$

As mentioned in No. $4 \mathrm{c}$ the equations of motion arise out of this.

5 b. Transition to the so-called Hamilton's principle. In a rather similar way to the well-known derivations in point mechanics, one can still transform d'Alembert's principle into other variational principles which determine the motion; here the point concerns in particular the transformation of the terms coming from the motion (the inertia forces) into the variation of a single expression being determined for every motion.

It is clear that, always using his Tacitean style, Hellinger greatly praises the contribution to continuum mechanics by D'Alembert, Lagrange and Hamilton. One has to notice that, unfortunately, Hellinger's opinion has been considered of minor importance or even explicitly wrong. Indeed, in [79] on p. 134, one can read:

«Granted its more modest scope, estimates of Lagrange's performance must remain a matter of taste. In music, in painting, in literature, tastes have changed in the past century. Why should they not also change in mechanics? The historians delight in repeating the Hamilton's praise of the Méchanique Analitique as "a kind of scientific poem", but it is unlikely that many persons today would find Hamilton's recommendations in non-scientific poetry congenial.»

Together with the authority of Hellinger, we believe instead that the ideas by Lagrange, Piola and Hamilton supply us a superior and more efficient logical tool which allows for easier formulation of more and more complex and general mathematical models of physical phenomena. Unfortunately, Lagrangian methods are mathematically sophisticated and only refined mathematical minds, like Hellinger's, are able to catch their more advanced subtleties. As we have already remarked in the first paper of this series, Hellinger is well aware of the concepts of Fréchet and Gâteaux derivatives of functionals. His updated competences in functional analysis are brilliantly used in the following sentences to confirm some results which had needed some involved reasoning when obtained by Lagrange himself. Remark that also in recent literature still the commutation rules of the variation delta and time derivatives are sometimes presented as a kind of magic trick. Note, on the contrary, how clearly and rigorously Hellinger proves the formula which follows below. However, differently from what is made too often in mathematical literature, Hellinger does not claim to have invented anything new, simply because he has obtained a well-known result in a more modern formalism: naturally and without any comment, he attributes the result to Lagrange, even indicating exactly the place where Lagrange, in his "Méchanique Analytique", obtains it.

16 If one wants to treat problems including nonholonomic constraints using d'Alembert's principle, then in the mechanics of continua, as in point mechanics, one has to refrain from considering that the variation of the motion for small $\sigma$ satisfies the constraints - moreover the constraint equations for the displacements are gained in a pure formal way by replacing the time differentiation by the operation $\delta$; cf. below p. 633 . Hereto one shall compare IV 1, No. 37, 38 (Voss) and the cited literature therein, in particular O. Hölder, Gött. Nachr., math.-phys. Kl. 1896, pp. 141-143, furthermore the presentations appeared so far from G. Hamel, Zeitschr. Math. Phys. 50 (1904), p. 1 and Math. Ann. 59 (1904), p. 416. 
Essential are, as in Lagrange ${ }^{17}$, the identities

$$
x^{\prime \prime} \delta x=\frac{d}{d t}\left(x^{\prime} \cdot \delta x\right)-\delta\left(\frac{1}{2} x^{2}\right) \quad(x, y, z),
$$

which follow from repeated differentiation of (6) from No. 2 with respect to the independent variables $\sigma, t$. By substituting that [relation] into (1) and taking into account, that the operation-symbols $\frac{d}{d t}$ and $\delta$ can be dragged, regardless of the factor $\varrho$, in front of the integral, since after the introduction of $a, b, c$ as variables of integration both the domain of integration $V_{0}$ and the remaining factor $\varrho_{0}$ are independent of $\sigma$ and $t$, we obtain

$$
-\frac{d}{d t} \iiint_{(V)} \varrho \sum_{(x y z)} x^{\prime} \delta x \cdot d V+\delta T+\delta A=0,
$$

where the abbreviation of the total kinetic energy

$$
T=\frac{1}{2} \iiint_{\left(V_{0}\right)} \varrho_{0} \sum_{(x y z)} x^{\prime 2} d V_{0}=\frac{1}{2} \iiint_{(V)} \varrho \sum_{(x y z)} x^{\prime 2} d V
$$

has been introduced. Eq. (4) is the equation used by G. Hamel ${ }^{18}$ and K. Heun ${ }^{19}$ under the name central equation of Lagrange as the foundation of mechanics of systems with finitely many degrees of freedom, which holds in the same manner also for the mechanics of continua ${ }^{20}$, and is completely equivalent to (1): the motion happens to be such that for every virtual displacement being admissible with respect to the possible constraints, the time derivative of the virtual work of the momentum $\left(x^{\prime}, y^{\prime}, z^{\prime}\right)$ per unit of mass is, at any instant, equal to the sum of the variation of the kinetic energy and the virtual work of all force effects. ${ }^{21}$

Considering now the motion within the time interval $t_{0} \leqq t \leqq t_{1}$, then (4) holds for every instant, and by integrating in $t$ with the boundaries $t_{0}, t_{1}$, it follows the so-called Hamilton's Principle when the virtual displacements are taken to be zero at the instants $t=t_{0}, t_{1}^{22}$ : By superimposing to the motion of the continuum virtual displacements admissible with respect to any possible conditions, which vanish without exception at the instants $\left[t_{0}\right.$ and $\left.t_{1}\right]$, then the time integral from $t_{0}$ to $t_{1}$ of the sum of the virtual work and the variation of the kinetic energy vanishes:

$$
\int_{t_{0}}^{t_{1}}(\delta T+\delta A) d t=0
$$

Since for every time interval in (6) the virtual displacements can be chosen arbitrarily, one can also easily imply the other way round that (4) or (1) follow from (6): these principles are completely equivalent.

Furthermore, one can now derive directly from these propositions the principle of least action in its various forms for the mechanics of continua ${ }^{23}$; nevertheless it seems - except for cases reducing to systems with finitely many degrees of freedom — that it has not found essential applications [up to now].

In the previous, sentences Hellinger proves how easy it is to generalize the principle of least action to continuum mechanics (and more generally in field theories). The applications of such principles are nowadays very important: for instance the great majority of numerical integration schemes are based on one of its formulations. It is astonishing how the simple and perfectly understood (by Hellinger and his cultural milieu) notions presented in the translated article may have been ignored or despised in such a large part of literature. In [79] on pp. 173-174, one can indeed read:

17 Mécan. anal., 2. part., sect. IV, art. 3.

18 G. Hamel, Zeitschr. Math. Phys. 50 (1904), p. 14.

19 K. Heun, Lehrbuch der Mechanik, T. 1: Kinematik (Leipzig 1906), p. 92. Cf. also IV 11, No. 11, K. Heun.

20 Cf. IV 11, No. 19 to 21 , K. Heun.

21 By varying also the time-parameter $t$, one can also obtain the relation denoted by G. Hamel (Math. Ann. 59 (1904), p. 423) and K. Heun ${ }^{19}$ as general central equation to the mechanics of continua; $c f . I V 11$, No. 19 to 21, K. Heun.

22 This principle has been formulated very soon by different authors for individual branches of the mechanics of continua, after one has got it for point mechanics (see IV 1, No. 42, Voss); one shall compare besides the literature of individual disciplines cited later on A. Walter, Anwendung der Methode Hamiltons auf die Grundgleichungen der math. Theorie der Elastizität, Diss. Berlin 1868, as well as the summarizing presentation in Kirchhoff's Mechanik, p. 117f. and W. Voigts Kompendium I, p. $227 \mathrm{ff}$.

23 For instance the considerations of O. Hölder, Die Prinzipien von Hamilton und Maupertius (Gött. Nachr., math.-phys. Kl. 1896, p. 122 ff.) can be extended immediately to continua. 


\begin{abstract}
«Almost as much nonsense has been written about the Méchanique Analitique as about the Principia and the Two New Sciences. Although Lagrange's book is far easier to read with understanding than is Newton's or Galileo's, still it does not seem to be easy enough for most historians of science to penetrate the contents. There are few errors, few novelties, and many routine manipulations in it. While it contains interesting historical parentheses, the presentation of mechanics is strictly algebraic, with no explanation of concepts, no illustrations either by diagrams or by developed examples, and no attempt to justify any limit process by rigorous mathematics. It does not enter at all a number of the fields opened by Newton, and it leaves unmentioned most of the deeper and harder problems of mechanics solved by the Basel geometers in the century preceding it. In particular, it does not include the general principle of moment of momentum. It could not do so, because the principle of virtual work does not yield the principle of moment of momentum until the nature of the contact forces is made somewhat explicit. Cauchy had still to be born and to create the general concept of stress, by which all theories of space-filling bodies are united.»
\end{abstract}

The future philologists (the reader is referred to the introduction) could have difficulties in framing the respective flourishing periods of Hellinger and Truesdell. Hellinger cites the works of Lagrange as if Lagrange had written them only few years before the article which we translate here. Hellinger treats his predecessors as if he were continuing their work exactly where they had left it: in this spirit he parallels Galileo who considers himself a disciple of Archimedes (see [68], p. 349) and behaves similarly as Piola, who few years after Lagrange dared to continue his work. Hellinger even does not remark that himself by using functional analytic tools can easily overcome some technical difficulties which were remarkable for Lagrange (see the previous commentary). He considers Lagrange to be such a giant that he treats as an unnoticeable detail his own superior technical capacity, correctly attributing it simply to a historical accident. In contrast, Truesdell proves to have an absolutely unjustified fixation for the law of balance of moment of momentum. He arrives to state that the «principle of virtual work does not yield the principle of moment of momentum» which is a flagrant fake as it was already known by Gabrio Piola, i.e. the successor of Lagrangian ideas in the context of continuum mechanics (see [18, 20,21]). It is also astonishing that in the work [82], which precedes [79] of about eight years, one finds on pp. 596-597 a theorem of Piola which states that the principle of virtual work is equivalent to Cauchy's first and second law (i.e. local balance of forces and local moment of momentum, respectively). We find such a statement somehow ambiguous: however, it proves that a textbook authored also by Truesdell contains a statement contradicting a statement found into another later textbook by Truesdell himself. The future philologists could be really puzzled by this circumstance: Nevertheless, we know [from a private communication with R. Toupin in 2013] that the part of the joint work [82] concerning variational principles was, in its more technical aspects, written by the youngest author. Also in the same joint work, Hellinger is cited only to be bitterly criticized (cf. footnote 1 on p. 595). The reader is referred again to the introduction: as the Mechanical Problems were transmitted to the posterity by attributing them to Aristotle, similarly it could be conjectured that Toupin managed to transmit in the community of continuum mechanics the basic concepts of variational principles allowing to his senior author to talk about them in a negative way.

Finally, it is not true that the concept of contact force cannot be developed without the logical procedure introduced by Cauchy with his tetrahedron argument: the reader is again referred to the works by Piola and the recent literature about them.

5c. The principle of least constraint. Also without the integration in time, one can transform the inertia terms in d'Alembert's principle into the variation of an expression determined for every motion by the state at the instant [of time] t only, in which certainly the appearance of time derivatives of second order must be allowed. In this way Gauss's principle of least constraint emerges ${ }^{24}$, which A. v. Brill has recently chosen as the starting point of a systematic treatment of the mechanics of continua. ${ }^{25}$

To obtain this principle, we take from the virtual displacements a family of varied motions No. 2, (6) of the following special kind: For the considered instant of time $t$, every particle $a, b, c$ shall have the same position and the same velocity as the actual motion, i. e. it shall hold for that very value $t$ :

$$
\delta x(a, b, c ; t)=0, \quad \delta x^{\prime}(a, b, c ; t)=0 \quad(x, y, z),
$$

while the variations $\delta x^{\prime \prime}, \delta y^{\prime \prime}, \delta z^{\prime \prime}$ of the accelerations are different from zero. One can now use these three functions as characteristic quantities of the displacements involved in (1). In the case of a freely deformable

24 Gauss' Werke V, p. 23 = Journal f. Math. 4 (1829). The first analytic formulation of this principle proposed by Gauss only verbally has been given by R. Lipschitz, Journ. f. Math. 82 (1877), p. 321 ff. and soon after by J. W. Gibbs, Amer. Journ. 2 (1879), p. $49=$ Scientif. Pap. II (New-York 1906), p. 1. For further literature see IV 1, No. 39, A. Voss.

25 A. v. Brill, Vorlesungen zur Einführung in die Mechanik raumerfüllender Massen, Leipzig 1909. 
continuum this is evident. However, when there is a condition of the form (3), two times differentiation with respect to t yields

$$
\sum_{(x y z)} \frac{\partial \omega}{\partial x} x^{\prime \prime}+\sum_{(x y z, a b c)} \frac{\partial \omega}{\partial x_{a}} x_{a}^{\prime \prime}+\cdots=0,
$$

where with the points some known functions $x, \ldots, x_{a}, \ldots$, and their first time derivatives are indicated. By the variation, i. e. differentiation with respect to $\sigma$, it follows due to (7) for the fixed instant $t$

$$
\sum_{(x y z)} \frac{\partial \omega}{\partial x} \delta x^{\prime \prime}+\sum_{(x y z, a b c)} \frac{\partial \omega}{\partial x_{a}} \delta x_{a}^{\prime \prime}=0,
$$

and this is in fact exactly condition $\left(3^{\prime}\right)$ for the $\delta x$ formulated above. Thus, the introduction of the functions $\delta x^{\prime \prime}, \ldots$ in (1) is allowed and after slight transformations the following new principle is obtained. ${ }^{26}$ ): Varying the actual motion of a continuum in a certain instant in such a way that the position and the velocity of every particle are conserved, but the acceleration is changed agreeing with the possible constraints, then the following sum of integrals always vanishes:

$$
\begin{aligned}
-\delta \iint_{(V)} \frac{1}{2} \varrho \sum_{(x y z)} x^{\prime \prime 2} d V+\iiint_{(V)}\left(\varrho \sum_{(X Y Z)} X \delta x^{\prime \prime}-\sum_{(X Y Z, x y z)} X_{x} \frac{\partial \delta x^{\prime \prime}}{\partial x}\right) d V & \\
& +\iint_{(S)} \sum_{(X Y Z)} \bar{X} \delta x^{\prime \prime} d S=0 .
\end{aligned}
$$

Instead of the variation of a quantity corresponding to an "averaged acceleration" appearing here ${ }^{27}$ one can also introduce the exact analogy of Gauss's constraint; then by attributing to the varied motion the same unchanged forces, one can write (8) as

$$
-\delta\left\{\iiint_{(V)} \frac{1}{2} \varrho \sum_{\left(\begin{array}{c}
x y z \\
X Y Z
\end{array}\right)}\left(x^{\prime \prime}-X\right)^{2} d V\right\}-\iiint_{(V)} \sum_{(X Y Z, x y z)} X_{x} \frac{\partial \delta x^{\prime \prime}}{\partial x} d V
$$

$$
+\iint_{(S)} \sum_{(X Y Z)} \bar{X} \delta x^{\prime \prime} d S=0
$$

The significant relevance of this principle lies, as in point mechanics, in the fact that it is valid completely unchanged for systems with nonholonomic constraints. For instance, [when] such a constraint equation, in which besides the functions of motion and their spatial derivatives also their first differential quotient with respect to time appear:

$$
\omega\left(a, b, c ; x, y, z ; x_{a}, \ldots, z_{c} ; x^{\prime}, y, z^{\prime} ; x_{a}^{\prime}, \ldots, z_{c}^{\prime} ; t\right)=0,
$$

then one obtains by once differentiating with respect to $t$ the condition the values of the acceleration [have to satisfy] for the fixed instant $t$, and by calculating the variation (differentiation with respect to $\sigma$ ) [subject to conditions] (7) it follows

$$
\sum_{(x y z)} \frac{\partial \omega}{\partial x^{\prime}} \delta x^{\prime \prime}+\sum_{(x y z, a b c)} \frac{\partial \omega}{\partial x_{a}^{\prime}} \delta x_{a}^{\prime \prime}=0,
$$

which henceforth has to be added to (8) as a constraint.

Especially, when $\omega$ is linear in the velocities $x^{\prime}, \ldots, x_{a}^{\prime}, \ldots$, then this result is, by its nature, identical to the form in which one frequently states d'Alembert's principle for nonholonomic conditions ${ }^{16}$ (Note of the translators: As in the original text, the text refers to the previous footnote 16), whereas the variations of the acceleration substitute the virtual displacements formally introduced therein.

An additional advantage of this principle in contrast to d'Alembert's [principle], which has hardly been used so far in the mechanics of continua, lies therein, that it provides a suitable basis for the treatment of kinetic problems with inequality constraints: one only has to ask expression (8) to be smaller or equal to zero for all

26 Brill, op. cit. p. $61 \mathrm{ff}$.

27 It has been used in this context at first by P. Appell, Paris C. R. 129 (1899), p. 317 and in a series of further works (s. IV 1, No. 38, Voss). 
variations of the acceleration which at an instant $t$ for a fixed position and velocity are admissible with respect to the constraints - exactly as it already has been stressed in particular by Gauss. ${ }^{28}$

For the elder author, this No. $5 \mathrm{c}$ is a golden nugget: it is unbelievable that the method conceived by Gauss (who was not a despicable mathematician, by the way) has not been exploited extensively up to now. The younger author as a pupil of Glocker, who extended the principle of least constraint in the context of rigid multibody systems subjected to setvalued force interactions [32,33], enjoyed this principle for finite dimensional systems already in his lectures on multibody dynamics. However, we have not found often in the literature its formulation (cf. also [63]) and we share with Hellinger the opinion that it seems to be very powerful and effective. We intend to investigate its uses already available in the literature and try to apply it to get effective integration codes to be used to predict the evolution of as many continuum systems as possible. Again, Hellinger seems to have done a very useful work for the community of mechanics, by making available in his entry of an Encyclopedia a contribution by Gauss not so easily available.

5d. Principles of general nature. To mention principles, which go beyond the so far discussed classical forms of the fundamental equations of kinetics, one must cite a generalization of Hamilton's principle, which plays quite similarly an important role in the dynamics of systems with finitely many degrees of freedom ${ }^{29}$; $\left[\right.$ the $^{2}$ generalization] lies therein, to use for the formation of the kinetic energy $T$ a more general function in the components of velocities, particularly a definite quadratic form: ${ }^{30}$ )

$$
T=\frac{1}{2} \iiint_{(V)} \mathfrak{T} d V, \text { where } \mathfrak{T}=\varrho_{11} x^{\prime 2}+2 \varrho_{12} x^{\prime} y^{\prime}+\cdots .
$$

Thereupon from Hamilton's principle (6) there follows equations of motion, which differ from (2) only in the point, that $\varrho \cdot x^{\prime \prime}, \ldots$ is substituted by $\frac{d}{d t}\left(\frac{\partial \mathfrak{T}}{\partial x^{\prime}}\right), \ldots$ The 6 coefficients $\varrho_{11}, \ldots$ are known functions of $a, b, c ;$ they determine together the density (mass of inertia) of the medium, which therefore depends on the direction (kinetic anisotropy).

Much further goes an another postulation, which makes on the particular form of the kinetic energy or the "inertia forces" due to the motion just as little assumptions, as the work expression in No. 3 on the nature of forces and stresses: One shall augment the principle of virtual displacements with a fourth independent variable - the time $t$ - [with] corresponding operations (integration in $t$ and addition of terms with time derivatives of $\delta x, \ldots)$ and [one shall] denote the fourfold integral, in which $a, b, c, t$ are considered to be independent, as virtual work of the moving continuum in the time interval $t_{0}, t_{1} .^{31}$

$$
\int_{t_{0}}^{t_{1}} d t \iiint_{\left(V_{0}\right)} d V_{0}\left(\sum_{\substack{x y z \\ X Y Z}}\left(\varrho_{0} X \delta x+X_{t} \frac{\partial \delta x}{\partial t}\right)-\sum_{\substack{x y z \\ X Y Z} ; a b c)} X_{a} \frac{\partial \delta x}{\partial a}\right) .
$$

Thereby, the components of momentum $X_{t}, \ldots$, representing the influence on the motion, shall be seen in the same way as the force and the stress quantities $X, \ldots, X_{a}, \ldots$ in their relation to the functions of motion according to the special nature of the continuum; one obtains the inertia forces commonly assumed so far, as (5), (6) show, for $X_{t}=\varrho_{0} x^{\prime}$, while (9) corresponds to a general linear ansatz in $x^{\prime}, y^{\prime}, z^{\prime}$. In addition, there can be added to (10) as in No. 3 similar integrals over the boundary of the domain of integration in the a-b-c-t-space. The motion now takes place in such a way, that the virtual work (10) vanishes for every infinitesimal virtual displacement being admissible with respect to the possible constraints; According to the well known methods, one can easily extract out of this the equations of motion - for instance for an arbitrarily continuously deformable continuum [it] follows:

$$
\frac{d X_{t}}{d t}=\varrho_{0} X+\frac{\partial X_{x}}{\partial a}+\frac{\partial X_{y}}{\partial b}+\frac{\partial X_{z}}{\partial c} \quad(X, Y, Z)
$$

28 Gauss, Werke V, p. 27.

29 Cf. IV 12, P. Stäckel

30 These approaches play an essential role in older optical theories of Lord Rayleigh; see especially Phil. Magaz. (4) 41 (1871), p. 519 (cf. V 21, No. 29, A. Wangerin). The same approach in T. J. Bromwich, Lond. math. Soc. Proc. 34 (1902), p. 307.

31 For the special case, that this virtual work is the variation of an "action integral", these approaches are formulated and pursued by E. and F. Cosserat, Corps déformables, p. 156ff. $(c f$. No. $7 \mathrm{~b})$. — In a form modified by the requirements of the theory of relativity the same approach appears in H. Minkowski, Grundgleichungen der elektromagnet. Vorgänge in bewegten Körpern, Gött. Nachr. 1908, p. 106 (cf. No. 16). 
and the boundary conditions are obtained analogously. Like in No. $3 c$ in (10), (11), one can introduce $x, y, z$ instead of $a, b, c$ as independent [variables $]^{32}$.

Also this section gives somehow the momentum to future investigations. It seems that the concepts presented here are of utility in the mechanics of continuum deformable porous media infused with compressible fluids. The interested reader is referred for instance to $[16,17,73]$ and references cited therein.

Completely analogously one has to extend the general kinetics of media with oriented particles considered in No. $4 b$, if one associates to these particles a resistance of inertia against angular accelerations: In order to formulate readily the most general expression, one has to add to (10) only the integral being analogous to No. 4 , $(2)^{33}$

$$
\int_{t_{0}}^{t_{1}} d t \iiint_{\left(V_{0}\right)} d V_{0}\left(\sum_{\substack{L M N \\ \pi \kappa e}}\left(\varrho_{0} L \delta \pi+L_{t} \frac{\partial \delta \pi}{\partial t}\right)-\sum_{\substack{L M N \\ \pi \kappa e} ; a b c)} L_{a} \frac{\partial \delta \pi}{\partial a}\right),
$$

where $L_{t}, \ldots$ determines the momentum of the internal rotation, and [one] can herefrom derive as in No. $4 \mathrm{~b}$ in every case the equations of motion, which have for a free movability of the triad a second triple [of equations] analogous to (11). ${ }^{34}$

All these considerations are with slight modifications also applicable for the dynamics of two- and onedimensional continua. ${ }^{35}$

\section{Annotated translation of No. 6 (pp. 637-643)}

\section{The forms of constitutive laws.}

\section{A. Formulation of general classes.}

6. The classes with dependence of the force effects on the deformation quantities. While in the previous discussions the effects - for the sake of brevity this expression includes besides forces and stresses of any kind also the momentum quantities of No. $5 d$ - have been considered in a mere formal way as coefficients of the virtual work expression, henceforth, [we] have to account for their connection with the characteristic quantities of the deformation or the motion of the continuum, which has to exist and has to be known, when after all the stated fundamental equations shall determine the deformation or the motion of the continuum. Moreover, [this connection] must express the clearly evident fact, that in every continuum due to motions and deformations certain force effects are induced, and that vice versa due to impressed forces and stresses motions and deformation are caused. Thereby primarily the [following] difference must be clarified, if the force effects are external, i. e. [the effects] have their cause in the relation to media and sources of effects located outside the considered medium (long-range forces, pressures at the boundary and such like), or internal, i.e. [the effects] are based on the material constitution of the particular medium and the mutual effects of the particles thereof. The last-named effects are for the objective at hand more essential; provided that the desired equations yield these [effects], they characterize the specific dynamic behavior of each one medium within the common classes of all continua and can consequently be denoted as material laws.

The previous sentences include a lucid distinction between internal and external work functional which has been often debated in the subsequent literature, where, more often, the article of Hellinger has not been well-understood or even simply ignored. To find a similarly clear presentation one has to wait, to our knowledge at least until Germain [27,28] or the very useful textbook of J. Salençon $[69,70]$. This clear set of statements make clear the role of constitutive theory in the mechanics of continua and reduces it to the most concise and effective formulation.

In the discussion, how these material laws are constituted in general, it is enough to refer primarily to the media treated in No. 3 and to the effective quantities of stress $X_{x}, \ldots, Z_{z}$ and if necessary to the force components

32 Cf. E. and F. Cosserat, op. cit. p. 187ff.

33 E. and F. Cosserat, op. cit. p. 156ff., p. $167 f f$.

34 Cf. also IV 11, No. 21c (K. Heun)

35 E. and F. Cosserat, op. cit. p. 121. The postulations regarding the kinetics of one-and two-dimensional continua can be based on those [used] in the statics of two- and three-dimensional [continua], respectively. 
$X, Y, Z$. Thereafter, the corresponding general schemes for the quantities of stress in the broader sense appearing in No. 4 and for the components of momentum of No. $5 \mathrm{~d}$ can be formulated easily - after all, the formulations [of the material laws] for these [effects] being effectively applied so far, can be deduced as special classes of dependence [which need] to be discussed in No. $7 b, f$.

The values of the stress components $X_{x}, \ldots, Z_{z}$ corresponding to the particle $a, b, c$ located at time $t$ at the position

$$
x=x(a, b, c ; t), \quad y=y(a, b, c ; t), \quad z=z(a, b, c ; t),
$$

must be given by the material laws for every possible motion of the continuum; hence [the values] are represented explicitly as expressions of any kind depending on $a, b, c, t$ and the functions (1). [These expressions] also include besides the values of the functions [(1)] and their spatial and time derivatives at the positions $a, b, c, t$ possibly values at other positions $\bar{a}, \bar{b}, \bar{c}, \bar{t}$ and in general the complete history in the domain of variability of the four variables (integrals and similar ones) - Hence, symbolically written in the form:

$$
F(a, b, c, t ; x(\bar{a}, \bar{b}, \bar{c}, \bar{t}), \ldots . .)
$$

Changing over to another orthogonal coordinate system $x, y, z$, then these nine expressions of the stress components have to be transformed like the components of a dyad (and similarly the expressions for $X, Y, Z$ like vector components and so on); if it concerns internal force effects, then there must exist equations between the transformed components and the new coordinates [which are] exactly of the old form.

This last sentence is a concise statement of the principle of objectivity (or principle of material frame-indifference) for constitutive equations for stress. The reader is invited to compare the present section by Hellinger to the statements found in Truesdell's First Course in Rational Mechanics [80]. Once the difference of notation is taken into account, the reader will remark the substantial coincidence of the content presented in both works - obviously the change of notations may make a content clearer but for sure it is not changing the attribution of scientific priority. In [80] (Chap. IV Constitutive Equations, Sect. 2 Constitutive Equations. Noll's Axioms) on p. 200, one reads: «The further development of continuum mechanics in this book will fall within the axioms laid down by Noll in $1958 . »{ }^{36}$ On p. 202, one finds the "Axiom N3. Principle of Material Frame-Indifference.", where capital N stands for Noll. The attentive reader will immediately remark that Truesdell claims that Noll has written in formulas exactly what Hellinger said in words. This transcription into formulas does not seem enough to attribute the Axiom to Noll. Consider that Hellinger finished his work in 1913 and did not attribute this axiom to himself. If one gives a glance to the historical overview about the principle of material-frame indifference in [81] and by considering Truesdell's awareness of Hellinger's article (cf. again footnote 1 on p. 595 of [82]), then Truesdell can be criticized in his own words found in [78] on p. 152: «Lagranges historische Angaben beziehen sich gewöhnlich auf die richtigen Quellen, verdrehen oder verringern jedoch ihren Inhalt.» ${ }^{37}$ Just as a side note, in the aforementioned textbook [80] published in 1977 which consists of 381 pages, Noll is cited (and highly praised as one of the main contributor to mechanical sciences) on 80 pages (often more than one time). Remark that instead Lagrange is cited only 12 times and mainly in a deprecative way. The strategy applied here was used in every era and cultural context and is really clear: a questionable historical overview together with almost excessive repetition will convince the Skeptical Scholars about the (desired) priority.

We consider now successively each argument possibly appearing in the expressions of stress; certainly, these effects which are discussed in the following individually can also appear simultaneously. Primarily, it has to be noted that the explicit appearance of the parameter $a, b, c$ indicates inhomogeneity, i. e. difference of the properties from particle to particle; [the] appearance of the time parameter indicates given external excitations, whose progress in time is a priori determined, irrespective of the actual occurring motion.

The bare essentials are certainly how the functions (1) themselves enter [in the functional dependence expressed by (2)] ${ }^{38}$; to begin with, we consider the case that [for the functions (1)] only their behavior in an arbitrary small vicinity of the position $a, b, c, t$, i.e. the values of the functions and their derivatives at this position, appear in (2), hence that (2) is of the form

$$
F\left(a, \ldots, t ; x(a, \ldots, t), \ldots ; x_{a}(a, \ldots, t), \ldots, x_{t}(a, \ldots, t) ; x_{a a}(a, \ldots, t), \ldots\right) .
$$

\footnotetext{
36 Noll 1958 corresponds to reference [52] in the paper at hand.

37 Most of [78], even in English, can be found in [79], including the hypothesis about Lagrange on p. 247: «Lagrange's histories usually give the right references but misrepresent or slight the contents.»

38 The classes of dependence, quoted in the following to begin with, have according to their form usually appeared at first in the development of the theory of elasticity; particulars are to be given in IIIB for the Nos. 9-16.
} 
The occurrence of local values of $x, y, z$ themselves corresponds with effects, which depend on the actual position of the individual particles in space, as they are for example external given force fields (gravity or similar ones). More characteristic for continua are however short-range effects, which manifest themselves in the appearance of stresses due to local deformations. As characteristic quantities of the whole deformation at a position (not only the pure shape change of the elementary theory of elasticity), one considers, as is well known, primarily the values of the nine first spatial derivatives thereof (cf. IV 14, No. 16); thus, the considered effect at hand expresses itself with an explicit dependence of the stress components on the values $x_{a}, \ldots, z_{c}$ at the position $a, b, c, t$. The type of these dependences must clarify, if and which individual elements of the deformation have exclusive or mainly influence on the stress or on the individual elements of the stress, as it will become clear later in the discussion of the particular fields.

The previous sentences lucidly formulate a property for constitutive equations which has solid physical grounds. Such a property is formulated in its essence already by Piola (cf. [20]) but named after Noll in some literature, [80].

The state of deformation at a position is described more precisely, if one uses besides the first also higher spatial derivatives of the functions (1), i. e. the deformation in the neighborhood is approximated by a transformation of higher order instead of a linear one; the dependence of the stresses on the deformation will be represented more completely, if one includes also these higher derivatives in the material laws. In fact, one has considered so far [derivatives which are] not higher than second derivatives, this is namely required not until then, when the state of the medium varies very quickly in space; the stresses at a position then depends also on the spatial slope of the common deformation quantities of 1 . order.

For the senior author, who cannot easily understand German, to read all previously mentioned sentences was rather astonishing. Indeed, because of the paper of Gurtin [34] and all papers influenced by it, for a long time it was believed, in a certain group of scientists and in a certain cultural milieu, that second (and a fortiori higher gradient materials) were NOT logically possible. In the aforementioned paper, one finds on p. 341 the following very clear statement: «One might ask the question: is it possible to have a material which obeys (1.6) but is not a simple elastic material? Here we prove that it is not.» What is astonishing is that the footnote 8 in the same paper WAS APPARENTLY not read by many followers of Gurtin (and sometimes one has the impression that Gurtin himself for a long period forgot his own footnote). This footnote reads:

«Thus the stress cannot depend upon the gradients of $\mathrm{F}$ and $\eta^{39}$. Of course this does not mean that higher order elasticity theories which include multipolar stresses are incorrect because of the dependence of these stresses on the higher order gradients. It simply means that one should not include such higher gradients if multipolar stresses are not included.»

After a long neglect of his own footnote, the late works of Gurtin came back to higher order gradient theories (see e.g. [26] and all related and subsequent papers), where the contribution by Toupin were reevaluated. It is remarkable that in the aforementioned paper a non-standard (sic!) principle of virtual work is formulated which can be found already in the present article and that (see the first paper of this series [23]) is proven by Hellinger himself to be exactly equivalent to the standard principle. We can formulate here a question which is related rather to psychology and history of science than to mechanics itself: how was it possible that Hellinger gave in 1913 as granted the possibility to introduce second gradient materials while still in 2016 many mechanicians believe that this possibility is forbidden by the second principle of thermodynamics? (recently also the younger author met the same difficulties which stopped the elder author some decades ago!). Nevertheless, there is also a large amount of papers available which prove the applicability of higher gradient theories, cf. for instance $[8,10,48,49,57,58,93,94]$. A very interesting field of applications for higher gradient theories lies in the description of pantographic structures, see [6,59,61,72,83-87] to mention just a few.

Equally to the local values of the spatial derivatives, in kinetics also the values of the time derivatives of the functions (1) at the position $a, b, c, t$ can enter in (3) explicitly; One has considered in particular the velocity components $x^{\prime}, y^{\prime}, z^{\prime}$ of the particles themselves and the "velocities of change" of the deformation quantities $x_{a}^{\prime}, \ldots, z_{c}^{\prime}-$ which, multiplied by $d t$, can also be interpreted as components of the deformation of the neighborhood of the particle $a, b, c$ due to the ongoing motion between $t$ and $t+d t^{40}$. These basic approaches satisfy the phenomena of external and internal friction, $i$. e. viscosity and similar ones.

39 With $F$ and $\eta$ the deformation gradient and the entropy are meant.

40 Stokes, Cambridge Phil. Trans. 8(1845) = Math. and Phys. Papers 1 (1880), p. 80; cf. also IV 15, No. 7, Love. 
For all laws of the class (3) the question, how these equations behave under a transformation of the directions of the $a$-b-c parameter lines through these points $a, b, c$, while the $x-y$-z-coordinates remain unchanged, is of fundamental evidence.

Here, Hellinger shows to be perfectly aware of the concept of material symmetry. Moreover, he clearly distinguishes between objectivity and material symmetry.

The attentive reader will also remark that in [80] the only references given to the Chap. IV Constitutive Relations are [9,52-54]. This observation, which although could be considered anecdotal, is indeed of great relevance at least for the scholars studying history of science and philology. Imagine that the often evoked Courageous Philologist will find only a part of nowadays literature: knowing how relevant the authority of Truesdell was, he could conclude that Noll was a giant of mechanics to whom one can attribute an enormous amount of results. He could courageously, but reasonably, state that probably the whole body of knowledge which we call continuum mechanics was formulated and advanced by this great scientist. However, the Skeptical Scholars will possibly have in their hands the article by Hellinger which we translate and comment here. These Scholars will need to fight a battle to state that:

i) Hellinger's article was preceding Noll's papers,

ii) the formalism used by Hellinger and Noll are equivalent,

iii) probably many experts of Linguistic will start to doubt about the meaning of the German words used by Hellinger to argue against the meaning to be given to his paper and its conceptual content.

What is unfortunately true and proven once more by the very particular history of continuum mechanics between 1822 (Piola's first work) and 1977 (the date of the aforementioned textbook [80]) is the following statement (see also Russo [68]). Science is not proceeding linearly. There are many steps ahead and many steps back: in general, more recent papers are NOT more advanced than elder ones. Also one unique hand may produce works having a not uniform scientific quality.

Thereby it is determined namely, if and which different directions through a point of the medium are tantamount for its constitution, provided that it is expressed in the considered material laws, i. e. it is decided on isotropy or aeolotropy of the medium; for specific conditions this connection has been studied thoroughly in the physics of crystals, where due to the mere restriction to infinitesimal deformation the difference between transformations of $a, b, c$ and $x, y, c$ does not appear. ${ }^{41}$

For the more general case, that within the material laws (2) also the values of the functions (1) at different positions and for different times enter, the characteristic ansatz is of sufficient generality - at first for statics to identify the components of stress with volume integrals over the whole continuum

$$
\iiint_{\left(V_{0}\right)} f\left(a, \ldots ; x, \ldots ; x_{a}, \ldots ; \bar{a}, \ldots ; \bar{x}, \ldots ; \bar{x}_{a}, \ldots\right) d \bar{a} d \bar{b} d \bar{c}
$$

whose integrands are given functions of the values of the deformation functions (1) and their derivatives for the particles $a, b, c$ and $\bar{a}, \bar{b}, \bar{c}$. Thereby long-range effects are included within the medium: an effect at the position $x, y, z$ emerges in consequence of the states at all other positions of the continuum. However, besides forces represented by basic approaches known from classical mechanics acting from mass particles to mass particles according to the class of forces of attraction, here it appears anew the long-range effects ("influence") considered by $P$. Duhem ${ }^{42}$, due to which at every position of the continuum superposed forces or stresses are caused by deformations taking place at all other positions of the continuum.

This statement proves that so called non-local continuum mechanics is formulated already in the works by Duhem. We managed to read only superficially the Duhem's second reference cited by Hellinger: it is however clear that Duhem develops (rather rigorously) a non-local continuum model for fluids which is compatible with thermodynamics. Duhem does not seem to be aware of the contribution by Gabrio Piola (see [14]). Hellinger's text indicates that the fundamental ideas on which peridynamics is founded were known also in the cultural milieu where Hellinger operated.

In the [field] of kinetics one will augment this ansatz such, that one adds a time integral over the whole motion; or rather - corresponding with our general notion of action and reaction - over the time before the

41 Cf. for instance F. Neumann, Vorles. üb. d. Theorie der Elastizität (Leipzig 1885), p. 164; W. Voigt, Abh. Ges. d. Wiss. Göttingen 34 (1887), 36 (1890), Kompendium I, p. $128 f f$. and p. 333, as well as in particular Lehrb. d. Krystallphysik (Leipzig 1910), \$286ff., \$ $370 f f ., ~ \$ 414 f f ., ~ \$ 462$.

42 P. Duhem, J. de math. (4) 8(1892), p. 311; Ann. de l'Éc. norm. (3) 10 (1893), p. 215, and 21 (1904), p. 117. 
considered instant $t$; thereby the integrand contains the values of the functions (1) as well as their derivatives at the instants $t$ and $\bar{t}(-\infty<\bar{t} \leqq t)$ :

$$
\int_{-\infty}^{t} d \bar{t} \iiint_{(V)} d \bar{a} d \bar{b} d \bar{c} f\left(a, \ldots, t ; x, \ldots, x_{a} ; \ldots, x_{t} ; \ldots ; \bar{a}, \ldots, \bar{t} ; \bar{x}, \ldots ; \bar{x}_{a} ; \ldots \bar{x}_{t} ; \ldots\right) .
$$

Originally, L. Boltzmann ${ }^{43}$ has used such expressions for the stress components to formulate the phenomenon of elastic residual effects, for which the stresses occurring in one instant depend in fact on all states the medium has passed in advance. Recently, V. Volterra ${ }^{44}$ has taken up the studies of problems arising from these integral formulations, once he has created with the theory of integro-differential equations a new tool for the analytical treatment thereof; by the way, he also allows in (5) multiple integrations with respect to time, where the integrands depend on the values of more than two instants of time. For all herein contained problems, for which the effects at one instant depend on the whole previous history of the system, he takes up the name "hereditary mechanics" introduced by E. Picard ${ }^{45}$.

By restricting oneself to analytic functions, then, under corresponding convergence requirements, one can replace the time integral (5) by a function of all (infinitely many) time derivatives of the functions $x, \ldots, x_{a}, \ldots$ at the instant of time $t$, as it is done by $W$. Voigt ${ }^{46}$ for the treatment of elastic residual effects.

We learn from Hellinger another precious historical circumstance: Boltzmann, Picard and Voigt had already started and developed the ideas put forward by Piola for founding non-local spatial continuum mechanics. Moreover, these authors also had started to study a version of continuum mechanics non-local in time.

Remark that at the end of [82] a quick reference to the same literature is given. The future Skeptical Scholars may use this circumstance to prove that Hellinger was a source for the first encyclopedic text by Truesdell, the one where, probably due to his co-author, at least some references to variational principles are found.

All these forms of material laws have been treated mainly for the special case that the deformations of the continuum are "infinitesimally small" (cf. IV 14, No. 16, Abraham). The functions (1) include this case, when one considers $a, b, c$ as spatial coordinates of the particle in the initial position and (cf. No. $2 a, p$. 607) by setting [these functions] with the help of a parameter $\sigma$, restricted to arbitrary small values, to:

$$
x(a, b, c ; t)=a+\sigma \cdot u(a, b, c ; t)+\cdots\left(\begin{array}{c}
x, y, z \\
u, v, w
\end{array}\right)
$$

where throughout the higher powers of $\sigma$ are neglected with respect to the lower ones. If an effect depends now on these functions of motion by a law of the form (3), then one has to replace the expression $F$ with the first terms of its expansion with respect to the powers of $\sigma$ due to (6); If the linear terms in $\sigma$ do not vanish identically, then one obtains as a result instead of (3) a law of the form:

$$
F+\sigma\left\{F_{x} \cdot u+\cdots+F_{x_{a}} \cdot u_{a}+\cdots+F_{x_{t}} \cdot u_{t}+\cdots+F_{x_{a a}} \cdot u_{a a}+\cdots\right\} .
$$

$F, F_{x}, \ldots$ are the values of the function (3) and its derivatives for $\sigma=0$, thus known functions of $a, b, c, t$; the material law is now linear in the local values of the functions $u(a, b, c ; t), \ldots$ determining the infinitesimal deformations, and the derivatives [of these functions] - according to Hooke's law of the theory of elasticity (cf. IV 23, No. 4). The term without $\sigma$ corresponds to initial forces or stresses, which can possibly exist in the undeformed continuum. Similarly, one could even consider stress laws, for which the coefficient of $\sigma^{1}$ vanishes $^{47}$; then for infinitesimally small deformations, the stresses would depend on the deformations at least quadratic contrary to Hooke's law, which consequently does not have to be valid necessarily even for infinitesimally small deformations.

In the previous paragraph, Hellinger accounts for the simplified scheme given by linearized continuum mechanics. In many cultural ambients, for some unclear reasons, linearized mechanics is considered "more intuitive". Somebody claims that

\footnotetext{
43 Wien. Ber. 70 (1874), p. 275 = Pogg. Annalen, Ergänzungsbd. 7 (1876), p. $624=$ Wissensch. Abh. I, p. 616.

44 The general fundamentals are included in Roma, Acc. Linc. Rend. (5) 18, 2 (1909), p. 295 and Acta math. 35 (1912), p. 295.

45 Riv. di Scienz. 1 (1907), p. 14.

46 Kompendium I, p. 458; cf. as well Cl. Maxwell, Scientif. Papers 2, p. 623.

47 With particular emphasis, B. de Saint-Venant has pointed this out in the discussion on the validity of Hooke's law; cf. his remarks in Navier, De la résistance des corps solides, $3^{\mathrm{e}}$ éd. (Paris 1864), p. 662 and Clebsch, Théorie de l'élasticité des corps solides (Paris 1883), p. 39.
} 
it is "more engineering". ${ }^{48}$ In reality linearizing an equation is a mathematical trick which aims to obtain solutions more easily. It happens more often that Linearized models have a unique solution and calculation techniques are more easily found. How it could happen that a choice based on purely mathematical reasons could become the "most" engineering one is another problem which deserves to be studied with rigor in the framework of the more general context of social sciences. Also in the linearized theories, Hellinger finds it natural to include second gradient materials. Furthermore, based on very clear considerations by Saint-Venant, Navier and Clebsch, he remarks that linearized models could NOT be valid also for small deformations, in some particular cases. The reader is referred, in this context, to the study of so-called statically undetermined structures, which cannot be studied in the framework of linearized models. Remark also that in the next paragraph Hellinger reaffirms the existence of higher gradient models.

If the material laws are of the integral form (4), (5), then the same considerations lead to a reduction of the integrand to a linear - certainly perhaps also to a higher [order] - function of the values of the displacements and their derivatives at the positions $a, b, c, t$ and $\bar{a}, \bar{b}, \bar{c}, \bar{t}$; for example, (4) becomes

$$
\iiint_{\left(V_{0}\right)}\left(f+\sigma\left\{f_{x} \cdot u+f_{x} \cdot \bar{u}+\cdots+f_{x_{a}} \cdot u_{a}+f_{\bar{x}_{a}} \cdot \bar{u}_{a}+\cdots\right\}\right) d \bar{a} d \bar{b} d \bar{c}
$$

and similarly the integral (5) simplifies for the case of temporal residual effects.

\section{Annotated translation of No. 7 (pp. 643-657)}

Hellinger has clearly in his mind the relationship between the principle of stationary action and the principle of virtual work. Indeed, once integrating also with respect to the time variable (and allowing for time dependent virtual displacements), it is easy to check that the formulation of the principle of virtual work is a more general form of the principle of stationary action, in which the dual quantities of velocities and deformation tensors are expressed in terms of the Lagrangian density of action. The class of theories encompassed in the principle of virtual work includes the class of theories which can be formulated with the principle of stationarity of action. The constitutive choice possible in the second case is limited to the choice of the action density, while in the first case one can choose with a constitutive equation all stresses and forces which are dual (see No. 4a in [23]) to all gradients of the fields $\delta x, \delta y, \delta z$. Therefore, the constitutive choices when using the stationary action principle are less rich than the constitutive choices available when using the principle of virtual work. This point seems not very clear in some literature. Indeed, in [26] we can read «Our goal here is to derive Toupin's results within a framework that is independent of constitutive equations. To do so we use a nonstandard form of the principle of virtual power [..]». This statement seems to imply that the novelties which the authors claim are present in their work consist in

i) a treatment which is independent of the constitutive equations - whatever this could mean,

ii) a nonstandard form of the principle of virtual power - this point was already discussed in the first paper [23] of this series.

Both the Courageous Philologist and the Skeptical Scholars eventually reading the paper [26] and the derived literature may be really puzzled. Whatever the expression "independent of the constitutive equation" may finally be interpreted, the anachronism with Hellinger's article and Piola's works could confuse completely those believing in the linear growth of knowledge and science. Only those who understood the ideas of Giambattista Vico will be able to eventually solve the puzzle: being however sharply criticized and attacked by orthodoxy. This potential confusion is proven, for instance, by the fact that one can read in [62] as criticized by [19]:

«Needless to say, the Principle is an invariant statement. The quantification on velocities is standard, that on body parts is not. Asking that (2) holds for all body parts is much stronger a requirement than demanding it to hold only for the whole body. ${ }^{1} »$

\footnotetext{
${ }^{1}$ We have been unable to assess who introduced this strengthened quantification in continuum mechanics first, when and where. Needless to say, in the absence of such a quantification, it would not be possible to characterize equilibrium for a system of rigid bodies, nor the method of Euler cuts would make any sense: $[\ldots]$
}

48 The elder author, when preparing his lecture in the national competition for associate professorship, was warned not to use non-linear deformation measures and to formulate the principle of virtual work only in the case of linear constitutive equations. The advice was intended to hide his mathematical background. Instead it seems clear that non-linear displacements are the only kind of displacements. 
Future philologists could argue that if authors mastering Italian language could not find in Piola one of the sources of the formulation of the principle of virtual work for every subbody of a given body, then most likely Piola is an author subsequent to Gurtin and Truesdell.

\section{Media with one characteristic state function.}

In the mechanics of continua particularly often media are considered whose characteristic equations can be reduced to one single function of the state variables. In case we talk at first about statics, such a reduction originates particularly from the assumption, that the virtual work coming into question is, up to sign, for every virtual displacement equivalent to the variation of a single scalar expression depending only on the corresponding state of deformation, [which is] the "potential" or the "potential energy" of the acting forces and stresses ${ }^{49}$; this assumption can be traced back to general theorems of thermodynamics.

The footnote 49 reflects clearly the opinion by Hellinger: Green has deduced for the first time the fundamental equations of elasticity using the principle of stationary action. Leaving aside the question of priority with Piola, we agree with Hellinger: it is most likely that new equations are derived for the first time by using variational principles. The reader is referred for instance to the discussions in Landau and Lifshitz [44] and in Feynman [24], Vol. II Sect. 12-1. Some further examples of situations where new models are introduced and motivated by means of variational principles are given in $[12,13,31,55,56,64]$. In this sense, there are many disciples of Hellinger all around.

7a. The common potential and its closest generalizations. The most simple form of this potential is characterized by the property that the potential of a domain dissected into parts is equal to the sum of the potentials $\Phi^{*}$ of the [corresponding] subdomains $V^{* 50}$. Under the obvious assumptions that $\Phi^{*}$ changes continuously with the boundary of $V^{*}$ and that the quotient $\Phi^{*}: V^{*}$ converges to a certain limit value $\bar{\varphi}$, when $V^{*}$ contracts around a certain point $\boldsymbol{x}, \boldsymbol{y}, \boldsymbol{z}$ indefinitely — and this regularly in the whole domain $V$ - it follows easily ${ }^{51}$ that the potential of the whole continuum $V$ (and similarly that of any subdomain) is represented by the volume integral, ranging over $V$, of the spatial function $\bar{\varphi}$ :

$$
\Phi=\iiint_{(V)} \bar{\varphi} d x d y d z=\iiint_{\left(V_{0}\right)} \varphi d a d b d c, \text { where } \varphi=\bar{\varphi} \frac{\partial(x, y, z)}{\partial(a, b, c)} .
$$

$\bar{\varphi}$ is the energy density per unit of volume of the deformed continuum, $\varphi$ is [the energy density] per unit of volume of the initial state; these are scalar quantities, which are for every continuum considered in a certain state of deformation continuous or yet piecewise continuous functions of $x, y, z$ and $a, b, c$, respectively. The nature of the continuum, independent of each of the occurring deformation, is determined when $\varphi$ is given as a function of the complete history of the deformation functions; if the dissection axiom shall hold for every deformation, then $\varphi$ can explicitly contain only the values of the functions and their derivatives [evaluated] at the considered position:

$$
\varphi=\varphi\left(a, b, c ; x(a, b, c), \ldots ; x_{a}(a, b, c), \ldots ; x_{a a}(a, b, c), \ldots\right)
$$

If it is about internal force effects, this function must be invariant with respect to orthogonal coordinate transformations in the $x-y$-z-space.

Hellinger introduces explicitly "the dissection axiom" in a way which seems to us much clearer (but equivalent) to Noll's(!?) axiom of local action (see p. 201, [80]). Once again, note that Hellinger considers energy densities depending on higher gradients and imposes objectivity on theses densities.

49 For simple cases already Lagrange has interpreted in the Méc. anal. such an assumption from the mechanics of discrete masses for continua (Prem. part., Sect, IV, No. 25) and applied it particularly in hydrostatics, by adding to the virtual work a term being proportional to the variation of the volume dilatation (1. part., sect VIII, No. 1); [this assumption] has undergone a further development in the theory of elasticity, namely G. Green (Cambr. Phil. Soc. Trans. 1838 = Math. Papers (London 1871), p. 245) has derived from it the fundamental equations for the first time. Cf. thereto IV 23, No. 5b

50 Already since the first direct introduction of the elastic potential, this assumption, as [being] natural, has been used more or less explicitly. A detailed explanation is given by P. Duhem, Le potential thermodynamique et la pression hydrostatique, Ann. Éc. Norm. (3) 10 (1893), p. 183.

51 Cf. P. Duhem, l. c., p. $187 \mathrm{ff}$. It is here merely a precise formulation of the process, common of old in mechanics, of the transformation of functions of a domain (as e.g. mass) into certain integrals. By the way, one needs to assume the uniform convergence of $\Phi^{*}: V^{*}$ only for a certain partition [which] in the limit tends to $V$ and can in addition certainly allow disconnections of the continuity and the uniform convergence at individual surfaces. 
At first we consider, that only the first derivatives appear. To find the connection with the effects ${ }^{52}$, we compute the potential of the varied deformation (No. 2a, (3)); Then the variation of $\Phi$ is obtained as

$$
\delta \Phi=\iiint_{\left(V_{0}\right)} \sum_{(x y z)}\left(\frac{\partial \varphi}{\partial x} \delta x+\frac{\partial \varphi}{\partial x_{a}} \delta x_{a}+\frac{\partial \varphi}{\partial x_{b}} \delta x_{b}+\frac{\partial \varphi}{\partial x_{c}} \delta x_{c}\right) d a d b d c
$$

where in the derivatives of $\varphi$ the unvaried values of $x, y, z$ and the derivatives thereof have to be inserted. From the identity

$$
\delta A=-\delta \Phi \quad \text { for all } \quad \delta x, \delta y, \delta z
$$

[and] by equating the coefficients of $\delta x, \ldots$ and the derivatives thereof, for a medium, which allows for all continuous virtual displacements, the material laws follow immediately. If one uses for $\delta A$ for instance the Ansatz No. 3c, (7), then ${ }^{53}$

$$
\varrho_{0} X=-\frac{\partial \varphi}{\partial x}, \quad X_{a}=\frac{\partial \varphi}{\partial x_{a}} \quad\left(\begin{array}{c}
x, y, z \\
X, Y, Z
\end{array} ; a, b, c\right)
$$

changing over to the quantities related to the deformed position by use of (8) from No. 3c and (1), then one obtains $^{54}: 55$

$$
\left\{\begin{array}{rll}
\varrho X & =-\frac{\partial \bar{\varphi}}{\partial x}, \\
X_{x} & =\frac{\partial \bar{\varphi}}{\partial x_{a}} \cdot x_{a}+\frac{\partial \bar{\varphi}}{\partial x_{b}} x_{b}+\frac{\partial \bar{\varphi}}{\partial x_{c}} x_{c}+\bar{\varphi}, & \\
X_{y} & =\frac{\partial \bar{\varphi}}{\partial x_{a}} y_{a}+\frac{\partial \bar{\varphi}}{\partial x_{b}} \cdot y_{b}+\frac{\partial \bar{\varphi}}{\partial x_{c}} \cdot y_{c}, & \\
X_{z} & =\frac{\partial \bar{\varphi}}{\partial x_{a}} z_{a}+\frac{\partial \bar{\varphi}}{\partial x_{b}} z_{b}+\frac{\partial \bar{\varphi}}{\partial x_{c}} z_{c} . &
\end{array}\right.
$$

Thereby, all of the considered material laws in No. 5 are reduced to the single equation (2), which gives $\varphi$ or $\bar{\varphi}$ as scalar functions of the local state of deformation.

In the previous paragraph, Hellinger writes in formulas what we announced in the commentary immediately after the title of Sect. 4. He shows the particular form of constitutive equations which have to be used in the principle of virtual work when actually the principle of stationary action is indeed valid. Hellinger gives us a careful account of the contributions to this part of continuum mechanics of which he is aware. He recognizes the contribution due to Lagrange concerning the general methods of the calculus of variations. Furthermore, he refers to Kirchhoff and attributes to him a variational approach to continuum mechanics, but Hellinger is wrong in attributing to Boussinesq the first representation of Eulerian components of stress as given by formula (5). Here, he seems to ignore that the transformations from Lagrangian to Eulerian description of the equilibrium conditions are (most likely) correctly to be attributed to Piola (see [14]). However, Hellinger is prudent. After the aforementioned attribution he wisely adds: By the way, in the theory of elasticity of finite deformations these formulas have been repeatedly derived and formulated anew. Both the future Courageous Philologist and the Skeptical Scholars will be completely confused in reading this text and in comparing it with Truesdell's ones. Indeed:

i) In [79] one can find a series of harsh negative evaluations about Lagrange's contribution to mechanical sciences.

ii) Piola is qualified by all sources and by himself as a follower of "our schoolmaster" the great Lagrangia (as Piola calls Lagrange, that is using his Italian family name).

iii) Truesdell attributes (p. 554 of [82]) to Piola the formula transforming Piola stress (the dual of the virtual displacement (velocity) gradient in material description, the quantities called $X_{a}$ etc. by Hellinger) into Cauchy stress.

iv) In [81], Piola is cited at least on 31 pages (many times several times on each page) in a very positive way (to be precise these citations nearly always concern one of the two Piola-Kirchhoff tensors).

52 Here, merely the approach common in the calculus of variations for the computation of the first variation for multiple integrals comes into consideration, as Lagrange (Misc. Taur. $2(1760 / 61)=$ Oeuvres 1, p. 353) originally has formulated it and [as he has] applied it in the Méc. anal. in many cases.

53 G. Kirchhoff, Sitzungsber. d. Akad. Wien, math.-nat., Kl. 9 (1852), p. 772.

54 J. Boussinesq, Mém. prés. p. div. sav., Paris 20 (1872), note 3. p. 591. Here only $\varphi$ instead of $\bar{\varphi}$ is used, but, what is essential, for the first time the components $X_{x}, \ldots$ instead of $X_{a}, \ldots$ are determined. By the way, in the theory of elasticity of finite deformations these formulas have been repeatedly derived and formulated anew.

55 Correction of the translators in the second equation of (5): The last addend $\bar{\varphi}$ seems to be a typo. 
v) Hellinger does not cite Piola in this context but cites Kirchhoff.

vi) In the subsequent paragraph Hellinger states how the presented treatment can be generalized to include higher gradients, but seems not to be aware of the contributions by Piola.

If the energy density (2) depends also on the second derivatives $x_{a a}, x_{a b}, \ldots$ of the deformation functions which in turn only comes into consideration for very quick changes in space of the state -, then new terms with second derivatives in the virtual displacements $\delta x_{a a}=\frac{\delta^{2} \delta x}{\delta a^{2}}, \ldots{ }^{56}$ will appear in $\delta \Phi$, and this results precisely in the in No. 4 a discussed additional terms to the original expression of the virtual work; thereupon both the components of this new effect, whose expressions emerge immediately from $\varphi$, and the old stress components, whose expressions have to be modified slightly, depend also on the second derivatives $x_{a a}, \ldots$

A special case, which can be classified here, shall be emphasized especially: namely, that to the potential (1) an integral over the surface of the continuum can be added:

$$
\Phi_{1}=\iint_{(S)} \bar{\psi} d S=\iint_{\left(S_{0}\right)} \psi d S_{0},
$$

where the "surface density" $\bar{\psi}$ or $\psi$ of the potential depends on the values at the surface $S$ of the deformation functions and the first derivatives thereof; such a potential can be characterized analogously to the foregoing axiom, [which] determines the form (1), that $\Phi_{1}^{*}: S^{*}$ approaches a finite value $\bar{\psi}$, when the surface element $S^{*}$ contracts around a point. In fact, one can transform (6) into a volume integral over $V$, if one adds second derivatives $x_{a a}, \ldots$. By the way, one can also compute $\delta \Phi_{1}$ directly and obtains then for the virtual work immediately a term of the form considered in No. $4 a$, (1).

The previous sentence describes a series of results which, in a completely analytical form, were later developed in [38]. Useless to say that an attentive reader may consider this last paper less original than what is claimed by its authors: actually its original result consists in the quantitative estimate of boundary conditions to be associated to second gradient continua in contact with an energetic material boundary. What had to be expected as a result is clearly described here by Hellinger. Remark that the following formula (6a) can also be found in [36].

If $\psi$ depends in particular only on the values of the deformation functions $x, y, z$ themselves, [and] not on the derivatives thereof, then $\delta \Phi_{1}$ has precisely the form of the work $\delta A_{3}$ of the compressive forces applied at the surface of the continuum (No. 3, (1)), and indeed their components become

$$
\bar{X}=\frac{\partial \bar{\psi}}{\partial x}, \quad \bar{Y}=\frac{\partial \bar{\psi}}{\partial y}, \quad \bar{Z}=\frac{\partial \bar{\psi}}{\partial z} .
$$

One can easily extend such potential-based approaches in such a way, that they yield force effects of the more general form (4) considered in No. 6. Thereto one only needs, according to the procedure of P. Duhem ${ }^{57}$, to substitute the axiom of the additive composition of the potential of the subdomains to the total potential by the assumption, that for a dissection of the continuum into $n$ subdomains $V_{1}, \ldots, V_{n}$, the potential $\Phi$ becomes a double sum

$$
\Phi=\sum_{i, k=1}^{n} \Phi_{i k}
$$

of $n^{2}$ summands, each of which $\Phi_{i k}$ depend only on the state of two subdomains $V_{i}, V_{k}$. By the application of similar continuity assumptions as mentioned above, $\Phi$ becomes equal to a sixfold integral, [which is] twice over $V$ or $V_{0}$, whose integrand depends on the values of the deformation functions and the derivatives thereof in two points $a, b, c$ and $\bar{a}, \bar{b}, \bar{c}{ }^{58}$ :

$$
\Phi=\iiint_{\left(V_{0}\right)} \iint_{\left(V_{0}\right)} \varphi\left(a, \ldots ; x, \ldots ; x_{a}, \ldots ; \bar{a}, \ldots ; \bar{x}, \ldots ; \bar{x}_{a}, \ldots\right) d a \ldots d \bar{c}
$$

56 There is a typo in the original source. The variation of the second spatial derivative writes commonly as $\delta x_{a a}=\frac{\partial^{2} \delta x}{\partial a^{2}}$.

57 P. Duhem, l. c., p. 188.

58 P. Duhem, l. c., p. 205. 
(In particular, when $\varphi$ includes a summand independent of the point $\bar{a}, \bar{b}, \bar{c}$, a summand of the form (1) can be included herein.) The variation of $\Phi$ becomes

$$
\left.\delta \Phi=\iiint_{\left(V_{0}\right)} \iint_{\left(V_{0}\right)} \int_{(x y z)} \sum_{(x y z ; a b c)}\left(\frac{\partial \varphi}{\partial x} \delta x+\frac{\partial \varphi}{\partial \bar{x}} \delta \bar{x}\right)+\sum_{(x y}\left(\frac{\partial \varphi}{\partial x_{a}} \delta x_{a}+\frac{\partial \varphi}{\partial \bar{x}_{a}} \delta \bar{x}_{a}\right)\right\} d a \ldots d \bar{c}
$$

and from the identity (3) [it] therefore follows for a medium, which allows for all continuous virtual displacements, the force and stress components at the point $a, b, c$ :

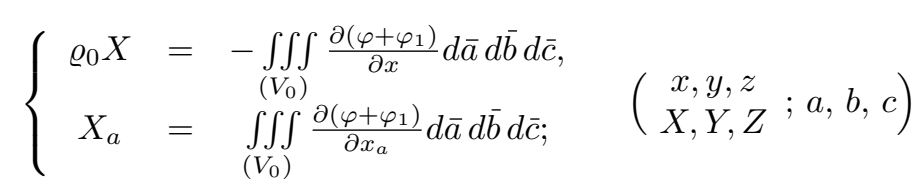

thereby $\varphi_{1}$ corresponds to the function arising from $\varphi$ by the permutation of the overlined and non-overlined arguments. As above the material laws, which express $X_{x}, \ldots$ by means of the one function $\varphi$, emerge directly out of this; P. Duhem has developed this [Ansatz] with respect to special assumptions corresponding with the circumstances of a pure theory of elasticity. ${ }^{59}$ Basically, there are approaches of this type, which are frequently used for the foundations of the mechanics of continua based on the perception of molecules. ${ }^{60}$ The double sums, which one formulates there for the potentials of the systems of molecules, become within the limit processes directly to integrals of type (7), and it is the question of the theory to formulate such assumptions, that for a correct guidance of the limit processes they transform into potentials of the simple forms (1) or (6); One confers for instance the presentation of H. Minkowski in V 9, No. 14.

In the previous paragraphs a quick presentation of the basic ideas of so called peridynamics are presented. In previous papers (see [14]), the credit as first founder of peridynamics is given to Piola. Hellinger credits Duhem and also claims that Duhem has found results to assure when peridynamics reduces to classical elasticity. Therefore not only in the Italian mechanical literature, but also in the German and French literature, peridynamics was known long before its modern formulation by Silling [75]. The historical analysis by Russo [68] applies precisely to this further case, in which there is no loss of sources: they are all easily available. It is therefore very difficult to claim that it is impossible to apply it to ancient Hellenistic sources: we claim that the continuous loss and rediscovery of scientific theories is such a common phenomenon that it continues to occur, EVEN WHEN SOURCES ARE NOT RARE OR LOST.

To be emphasized particularly is again the formulation of the potential-based approaches for the case of "infinitesimal" deformation of the continuum (No. 6, (6)). By neglecting the quadratic terms in $\sigma^{61}$, the expressions of force and stress components turn according to (4) into

$$
\varrho_{0} X=-\frac{1}{\sigma} \frac{\partial \tilde{\varphi}}{\partial u}, \quad X_{a}=-\frac{1}{\sigma} \frac{\partial \tilde{\varphi}}{\partial u_{a}}, \quad\left(\begin{array}{c}
u, v, w \\
X, Y, Z
\end{array} ; a, b, c\right)
$$

thereby $\tilde{\varphi}$ corresponds to those terms of the power series of the energy density $\varphi$ being linear and quadratic in $\sigma$, which arise from the terms linear in $\sigma$ of the series (6) of No. 6 :

$$
\begin{aligned}
\tilde{\varphi}=\varphi^{0}+\sigma\left(\varphi_{x}^{0} u\right. & \left.+\cdots+\varphi_{x_{a}}^{0} u_{a}+\cdots\right) \\
& +\frac{\sigma^{2}}{2}\left(\varphi_{x x}^{0} u^{2}+\varphi_{x y}^{0} u v+\cdots+\varphi_{x x_{a}}^{0} u u_{a}+\cdots+\varphi_{x_{a} x_{a}}^{0} u_{a}^{2}+\varphi_{x_{a} x_{b}}^{0} u_{a} u_{b}+\cdots\right)
\end{aligned}
$$

wherein the derivatives of $\varphi$ signed with the label 0 have to be evaluated for $\sigma=0$, i. e. for the arguments $x=a, \ldots, x_{a}=1, x_{b}=0, \ldots$. The expressions (9a) are in fact of the class of Hooke's law considered in No. 6 , $\left(3^{\prime}\right)$; thereby [it] is naturally required, that the terms of the expansion of $\varphi$ constituting $\tilde{\varphi}$ do not vanish identically. The stress components with respect to the deformed position of the continuum $X_{x}, \ldots$ differ according to No. $3 c$, (8) from $X_{a}, \ldots$ by the following expressions linear in $\sigma$ :

$$
X_{x}-X_{a}=\sigma\left(-\varphi_{x_{a}}^{0}\left(v_{b}+w_{c}\right)+\varphi_{x_{b}}^{0} u_{b}+\varphi_{x_{c}}^{0} u_{c}\right), \ldots
$$

59 P. Duhem Ann. Éc. Norm., (3) 21 (1904), p. 117ff. Also separately: Récherche sur l'élasticité, Paris 1906.

60 E. g. in Navier's theory of the elastic potential (cf. IV 23, No. 5a, Müller-Timpe) and in the theory of capillarity of P. S. Laplace and C. Fr. Gauss (cf. V9, No. 13, Minkowski).

61 H. Poincaré, Lecons sur la théorie de l'Élasticité, Paris 1892, p. 54ff.; E. and F. Cosserat, Ann. de la Fac. des Sciences de Toulouse 10 (l896), p. J. $70 f f$. 
and these become only zero or of the order of magnitude $\sigma^{2}$ of the otherwise neglected quantities, when the "initial stresses" given by $\varphi_{x_{a}}^{0}$ vanish before the infinitesimal deformation. ${ }^{62}$ - Thereafter no more detailed presentation is required, how one reformulates in a similar way the more general potential-based approach of Duhem (7) for infinitesimal deformations.

The previous paragraphs describe the linearization process in the case of conservative continua. The treatment is standard: the reader will remark that here the choice to avoid Levi-Civita notation makes the presentation rather cumbersome and that the consideration of initial stresses is rather quick and maybe too synthetic.

\section{7b. The potential-based approach for media with oriented particles.}

The works by the Cosserat brothers have been intensively and carefully studied since their first appearance and Hellinger is carefully reporting their results in this section. One remark is appropriate here. In the previous sections, Hellinger did not introduce yet any "measure of deformation" for first and second gradient (or higher gradient) continua: we will find a detailed description for these measures later in the presently translated article. Moreover in the subsequent p. 686, Hellinger states:

«It is said briefly, that the entire physics is invariant with respect to the group of all orthogonal coordinate transformations of the ordinary geometry, the so-called "basic group" or "Euclidean group". Herefrom it follows in particular, that the virtual work of all internal effects within a continuous system necessarily vanishes for a virtual displacement corresponding to an infinitesimal change of the coordinate system, or that the total potential of these effects remain unchanged for any such displacement of the continuum, i.e. [that the potential] is a euclidean potential in the sense of E. and F. Cosserat (cf. No. 7b, p. 650).»

In [18] it is shown how Gabrio Piola (see also e.g. [14]) had examined carefully the consequences of the invariance under superimposed rigid motions on constitutive equations for stresses and managed to characterize the whole set of deformation measures for $\mathrm{N}$-th gradient continua (i.e. continua whose internal work depends on all gradients of the placement up to the order $\mathrm{N}$ ). Here, Hellinger introduces very carefully the deformation measure for Cosserat continua and it is clear that the sources he could master are much more detailed in this context than those concerning Piola. Most probably, Piola's textbook reached Hellinger only via indirect citations ${ }^{63}$ or possibly Hellinger could read French much better than Italian. The future Skeptical Scholars are warned: Piola did introduce the correct deformation measures for continua whose kinematics is characterized by the placement field only and Piola is antecedent the Cosserat brothers. Hellinger is aware of the need to impose to the internal work functionals some invariance requirements and states that these functionals must be invariant. He does report in his article the analytical results obtained by Piola for this characterization (see for instance the Nos. 9 and 12 to be translated in the next paper of this series) but he neither attribute these results to Piola nor cites Piola's works, which contain fundamental results in this context.

According to the procedure of E. and F. Cosserat ${ }^{64}$ one can extend this potential-based approach also to continua, whose particles are endowed with a certain orientation; one only has to assume, that the energy density $\varphi$, usually defined as in No. 7a, depends besides the so far considered quantities also on the parameters $\lambda, \mu, \nu,[w h i c h]$ determine the actual orientation of the particle $a, b, c($ No. $2 b,(9))$, and the (first) derivatives with respect to $a, b, c$ of these [parameters]:

$$
\varphi=\varphi\left(\lambda(a, b, c), \ldots ; \lambda_{a}(a, b, c), \ldots, \nu_{c}(a, b, c)\right) .
$$

A virtual rotation of the individual particles No. 2 (10) then yields the following contribution to the variation of the potential:

$$
\delta \Phi=\iiint_{\left(V_{0}\right)} \sum_{(\lambda \mu \nu)}\left(\frac{\partial \varphi}{\partial \lambda} \delta \lambda+\frac{\partial \varphi}{\partial \lambda_{a}} \delta \lambda_{a}+\frac{\partial \varphi}{\partial \lambda_{b}} \delta \lambda_{b}+\frac{\partial \varphi}{\partial \lambda_{c}} \delta \lambda_{c}\right) d a d b d c .
$$

If one introduces now due to No. 2, (11), (12) the angular velocities $\delta \pi, \delta \kappa, \delta \varrho$ of the virtual rotation and by considering that

$$
\delta \lambda_{a}=\frac{\partial \delta \lambda}{\partial a}=\sum_{\substack{l m n \\
\pi \kappa \varrho}}\left(\frac{\partial l_{1}}{\partial a} \delta \pi+l_{1} \frac{\partial \delta \pi}{\partial a}\right) \quad\left(\begin{array}{c}
\lambda, \mu, \nu \\
1,2,3
\end{array} ; a, b, c\right),
$$

62 J. Boussinesq, op. cit. ${ }^{54}$, p. 598, E. and F. Cosserat, l. c., p. J. 74 f.

63 Hellinger's reference to Piola in No. 3d, see [23], implies that his source is Müller and Timpe [51], where Piola's ansatz is described briefly.

64 E. and F. Cosserat, "Corps déformables" 5 ), chap. IV, p. $122 \mathrm{ff}$. 
then, by identification of $-\delta \Phi$ with No. 4, (2) and $\left(2^{\prime}\right)$, respectively, the following formulas for the torques acting at the mass and surface elements emerge ${ }^{65}$ :

$$
\left\{\begin{aligned}
\varrho_{0} L & =-\sum_{\left(\begin{array}{l}
\lambda \mu \nu \\
123
\end{array}\right)}\left\{\frac{\partial \varphi}{\partial \lambda} \cdot l_{1}+\frac{\partial \varphi}{\partial \lambda_{a}} \frac{\partial l_{1}}{\partial a}+\frac{\partial \varphi}{\partial \lambda_{b}} \frac{\partial l_{1}}{\partial b}+\frac{\partial \varphi}{\partial \lambda_{c}} \frac{\partial l_{1}}{\partial c}\right\}, \\
L_{a} & =\frac{\partial \varphi}{\partial \lambda_{a}} l_{1}+\frac{\partial \varphi}{\partial \mu_{a}} l_{2}+\frac{\partial \varphi}{\partial \nu_{a}} l_{3} .
\end{aligned} \quad\left(\begin{array}{c}
L, M, N \\
l, m, n
\end{array} ; a, b, c\right)\right.
$$

The footnote 65 is really interesting. Here, Hellinger writes the stationarity condition for the action of the Cosserat continua WITHOUT requiring any invariance condition for the deformation energy and without introducing any objective deformation measure. Anybody who had to develop a numerical code for studying the deformation of any kind of body knows how useful these equations may be. The invariance must be most suitably introduced in subsequent developments of the theory. Exactly as suggested by Hellinger. For more recent applications and developments in the theory of generalized continua we refer to $[25,60,65,77,88]$.

Often it is useful to introduce in these formulas the angular velocities analogously to the $\delta \pi, \delta \kappa, \delta \varrho$, which appear in the transition from one triad of a particle to the one of the neighboring particle; we consider especially the neighboring particles in direction of the parameter lines $a, b, c, i . e$. the components of the angular velocities

$$
p_{a}=\sum_{(123)} \beta_{1} \frac{\partial \gamma_{1}}{\partial a}, q_{a}=\sum_{(123)} \gamma_{1} \frac{\partial \alpha_{1}}{\partial a}, r_{a}=\sum_{(123)} \alpha_{1} \frac{\partial \beta_{1}}{\partial a} \quad(a, b, c)
$$

Then one has analogously to the relation (12) of No. 2

$$
\frac{\partial \lambda}{\partial a}=l_{1} p_{a}+m_{1} q_{a}+n_{1} r_{a} \quad\left(\begin{array}{c}
\lambda, \mu, \nu \\
1,2,3
\end{array} ; a, b, c\right)
$$

and [one] can substitute the $\lambda_{a}, \ldots, \nu_{c}$ with the angular velocities $p_{a}, \ldots, r_{c}$ in the expression (11) of the energy density:

$$
\varphi=\varphi\left(\lambda, \mu, \nu ; p_{a}, p_{b}, \ldots, r_{c}\right)
$$

If one computes $\delta \Phi$ with this expression and by considering the relation following from (13) (the analogue to the so called "transition equations" 66 of kinetics)

$$
\delta p_{a}=\frac{\partial \delta \pi}{\partial a}+r_{a} \delta \kappa-q_{a} \delta \varrho \quad\left(\begin{array}{c}
p, q, r \\
\pi, \kappa, \varrho
\end{array} ; a, b, c\right),
$$

then similar considerations which lead to $(12)^{65}$ (Note of the translators: As in the original text, the text refers to the previous footnote 65 ) result in:

$$
\left\{\begin{aligned}
\varrho_{0} L & =-\left\{\frac{\partial \varphi}{\partial \lambda} l_{1}+\frac{\partial \varphi}{\partial \mu} l_{2}+\frac{\partial \varphi}{\partial \nu} l_{3}+\sum_{(a b c)}\left(q_{a} \frac{\partial \varphi}{\partial r_{a}}-r_{a} \frac{\partial \varphi}{\partial q_{a}}\right)\right\}\left(\begin{array}{c}
L, M, N \\
l, m, n
\end{array}\right) \\
L_{a}= & \left.\frac{\partial \varphi}{\partial p_{a}} . \begin{array}{c}
L, M, N ; a, b, c) \\
p, q, r
\end{array} ; a, b\right)
\end{aligned}\right.
$$

Using No. 4b, (5), the transformation to the components of the torques with respect to the deformed continuum $L_{x}, \ldots, N_{z}$ can be carried out easily.

$E$. and F. Cosserat considered in particular the internal actions in a medium represented by this ansatz[.] For these [actions] $\varphi$ is, as a function of $x, \ldots$ and $\lambda, \ldots$, invariant with respect to orthogonal coordinate transformations in the $x-y$-z-space, or - what implies the same - these [actions for which] every motion of the continuum together with the adjoint triads being regarded as rigid leaves the potential unchanged; they call such a potential a euclidean one (action Euclidienne). To describe this class of potentials, they use in every point $x, y, z$ as (moving) frame of reference the actual position of the triad attached to the particle just located

65 These formulas cannot be found explicitly in the book of the Cosserats, since therein the assumption of a "Euclidean" potential, which is achieved below, forms the basis; however, they are contained in the equations of p. $132 \mathrm{ff}$. and 141 or p. $130 \mathrm{ff}$. and 134ff.; The identification occurs easiest starting from the formulas for the work given on $p .138 f f$.

66 They change into these [equations], when a is replaced by a time parameter; cf. IV 6 (P. Stäckel), No. 30, p. 584f. and remark ${ }^{417}$ ) as well as IV 11 (K. Heun), No. $14 c$. 
there; The components $p_{a}, \ldots, r_{c}$ are substituted by the components of the same angular velocities formulated with respect to these new axes:

$$
\mathfrak{p}_{a}=\alpha_{1} p_{a}+\beta_{1} q_{a}+\gamma_{1} r_{a}=\alpha_{3} \frac{\partial \alpha_{2}}{\partial \alpha}+\beta_{3} \frac{\partial \beta_{2}}{\partial \alpha}+\gamma_{3} \frac{\partial \gamma_{2}}{\partial \alpha}\left(\begin{array}{c}
\mathfrak{p}, \mathfrak{q}, \mathfrak{r} \\
1,2,3
\end{array} ; a, b, c\right),
$$

and in a similar way the 9 deformation quantities $x_{a}, \ldots, z_{c}$ shall be transformed to:

$$
\mathfrak{x}_{a}=\alpha_{1} x_{a}+\beta_{1} y_{a}+\gamma_{1} z_{a} \quad\left(\begin{array}{l}
\mathfrak{x}, \mathfrak{y}, \mathfrak{z} \\
1,2,3
\end{array} ; a, b, c\right) .
$$

Then the most general euclidean potential, which depends at most on the first derivatives of the deformation functions, is an arbitrary function of these 18 quantities $\mathfrak{p}_{a}, \ldots \mathfrak{z} c$, [functions] which moreover can explicitly contain $a, b, c^{67}$ :

$$
\varphi=\varphi\left(a, \ldots ; \mathfrak{x}_{a}, \ldots, \mathfrak{z}_{c} ; \mathfrak{p}_{a}, \ldots, \mathfrak{r}_{c}\right)
$$

For the derivation of the equilibrium conditions for this ansatz one also introduces the components of the virtual displacement and rotation with respect to the new moving axes:

$$
\begin{aligned}
\delta \mathfrak{x} & =\alpha_{1} \delta x+\beta_{1} \delta y+\gamma_{1} \delta z, \\
\delta \mathfrak{i} & =\alpha_{1} \delta \pi+\beta_{1} \delta \kappa+\gamma_{1} \delta \varrho ;
\end{aligned} \quad\left(\begin{array}{l}
\mathfrak{x}, \mathfrak{y}, \mathfrak{z} \\
\mathfrak{i}, \mathfrak{j}, \mathfrak{k} \\
1,2,3
\end{array}\right)
$$

then one has the "transition equations"

$$
\begin{aligned}
& \delta \mathfrak{x}_{a}=\frac{\partial \delta \mathfrak{x}}{\partial a}+\mathfrak{q}_{a} \delta \mathfrak{z}-\mathfrak{r}_{a} \delta \mathfrak{y}_{a} \delta \mathfrak{k}-\mathfrak{z}_{a} \delta \mathfrak{j}, \\
& \delta \mathfrak{p}_{a}=\frac{\partial \delta \mathfrak{i}}{\partial a}+\mathfrak{q}_{a} \delta \mathfrak{k}-\mathfrak{r}_{a} \delta \mathfrak{j} \\
& \left(\begin{array}{l}
\mathfrak{x}, \mathfrak{y}, \mathfrak{z} \\
\mathfrak{p}, \mathfrak{q}, \mathfrak{r} ; a, b, c) \\
\mathfrak{i}, \mathfrak{j}, \mathfrak{k}
\end{array}\right)
\end{aligned}
$$

and is therefore immediately able to compare the variation of the potential formulated with (17)

$$
\left.\delta \Phi=\iiint_{\left(V_{0}\right)} \sum_{\substack{\mathfrak{x}, \mathfrak{y}, \mathfrak{z} \\ \mathfrak{p}, \mathfrak{q}, \mathfrak{r}}} ; a, b, c\right)\left(\frac{\partial \varphi}{\partial \mathfrak{x}_{a}} \delta \mathfrak{x}_{a}+\frac{\partial \varphi}{\partial \mathfrak{p}_{a}} \delta \mathfrak{p}_{a}\right) d a d b d c
$$

with the following form of the virtual work:

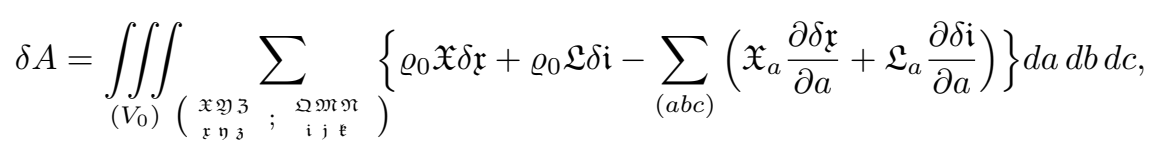

in which the components of the earlier considered forces, stresses and torques appear with resepct to the moving coordinate triad. Accordingly, this results in formulas ${ }^{68}$ of the type

$$
\left\{\begin{array}{l}
\varrho_{0} \mathfrak{X}=\sum_{(a b c)}\left(\mathfrak{r}_{a} \frac{\partial \varphi}{\partial \mathfrak{y}_{a}}-\mathfrak{q}_{a} \frac{\partial \varphi}{\partial \mathfrak{y}_{a}}\right), \quad \mathfrak{X}_{a}=\frac{\partial \varphi}{\partial \mathfrak{x}_{a}} \\
\varrho_{0} \mathfrak{L}=\sum_{(a b c)}\left(\mathfrak{r}_{a} \frac{\partial \varphi}{\partial \mathfrak{q}_{a}}-\mathfrak{q}_{a} \frac{\partial \varphi}{\partial \mathfrak{r}_{a}}+\mathfrak{z} a \frac{\partial \varphi}{\partial \mathfrak{y}_{a}}-\mathfrak{y}_{a} \frac{\partial \varphi}{\partial \mathfrak{z}_{a}}\right), \quad \mathfrak{L}_{a}=\frac{\partial \varphi}{\partial \mathfrak{p}_{a}} .
\end{array}\right.
$$

\section{7c. The potential-based approach for two- and one-dimensional continua.}

In this section, Hellinger sketches in a few sentences the most effective procedure to obtain from a three-dimensional continuum model a reduced continuum model of lower dimensions - although still moving in three-dimensional space. He considers as natural the introduction of lower dimensional Cosserat continua by starting (eventually) from a threedimensional Cosserat continuum. The attentive reader will remark that Hellinger considers as obvious that the best way to proceed is the following: one must write the energy for lower dimensional continua in terms of the energy of the threedimensional continuum to be reduced, once an integration with respect to the "smaller" dimensions is performed. Not much

67 E. and F. Cosserat, "Corps déformables”, p. 127.

68 E. and F. Cosserat, l. c., p. 130f.; cf. also IV 11 K. Heun No. 21. 
details are given so that one cannot guess how far Hellinger was from the concept of Gamma-convergence. However, the idea of the identification of energies arising in different models is there. Moreover, the procedure of Piola to get macromodels from micro-models (what we try to call Piola's Ansatz, see e.g. [15]) was formulated before Hellinger and used in many contexts. As future Courageous Philologists, we can conjecture that Hellinger was at least aware of the heuristic importance of the homogenization procedure via identification of energies. Indeed, Hellinger:

i) was a great expert and one of the founders of modern functional analysis,

ii) quotes the available results in this context by Lagrange,

iii) was able to distinguish two different mathematical models for one single physical object due to his epistemological understanding of mechanics.

Anyway, the reader is referred to the next sections for further considerations about the last point.

For the two- and one-dimensional extended continua in the tree-dimensional space, one can gain the potentialbased approach without any difficulties using rather similar considerations. ${ }^{69}$ The energy density $\varphi-$ as the assumed existing limit of the quotient between the potential of a part of the continuum becoming continuously smaller and the area or length thereof - becomes a given function of the 6 functions $x, y, z, \lambda, \mu, \nu$ of $a, b$ (or of a) and the derivatives thereof, the potential itself [becomes] consequently a two-or one-dimensional integral:

$$
\Phi=\iint_{\left(S_{0}\right)} \varphi d a d b \quad \text { or } \Phi=\int_{0}^{l} \varphi d a .
$$

The variation of these potentials and therefore the force, stress and torque components formulated with respect to the initial parameters are obtained immediately from the corresponding formulas of the three-dimensional case by omitting the terms concerning $c$ or $b$ and $c$; The transformation to the quantities formulated with respect to the deformed state follows then according to No. $3 e$, (16) and No. 4b, (12).

If one focuses on oriented particles, then the angular velocities $p_{a}, \ldots$ as defined in No. $7 b$ play again a crucial role, and indeed one has now naturally only 2 or 1 triple of these quantities. E. and F. Cosserat have widely developed the theory of such media using the triad associated with every particle as moving frame of reference $e^{70}$ and have considered also here the internal actions, which are derived from a euclidean potential as defined above; the expression of this potential and the corresponding force, stress and torque formulas are again obtained by the specialization of the equations (16) ff. of No. $7 \mathrm{~b}$.

$7 d$. The relevance of the effective minimum. An essential advantage of the existence of a potential $\Phi$ of the total force effects is the possibility to express the equilibrium conditions without explicitly using infinitesimal displacements. The equilibrium condition $\delta \Phi=0$ is namely the condition that $\Phi$ has for the considered deformation an extremum (maximum or minimum, but possibly also a so called "saddle point") ${ }^{71}$ : For an equilibrium position of the portion $V_{0}$ of the continuum, the potential of $V_{0}$ therefore becomes an extremum (in the broadest sense), compared with the values for all neighboring states of deformations admissible with respect to possibly occurring constraints.

Here, Hellinger continues to use the authority of Lagrange to show the features of the presented theories. In particular, he refers the visionary understanding by Lagrange of a circumstance which has been exploited systematically in modern computational mechanics: one can characterize equilibrium configurations of INFINITE DIMENSIONAL continuous systems via the search of a MINIMA of functionals. Hellinger contradicts here the future commentator (i.e. Truesdell) who sharply criticizes Lagrange in this aspect. Indeed in the preface of [80] one finds the following statements.

«The mechanics of finite systems of points and rigid bodies was given a fairly definitive form by Lagrange's exposition in his Méchanique Analitique, 1788. While that book covers only certain aspects of the rational mechanics created by Lagrange's great predecessors,»

69 Already Lagrange applied [the ansatz] to the problems in this area which he considered ${ }^{23}$ ) (Note of the translators: This corresponds to the footnote 39 in [23], i. e. «Mécan. anal., 1. part., sect. IV. § II, as well as for a series of particular problems in sect. V-VIII.») ; [the ansatz] was developed further in the theory of elastic wires and plates (cf. IV 6 (P. Stäckel), No. 23, 24 and IV 25, Kap. III, Tedone-Timpe), but particularly also in the theory of capillarity (cf. V9 (Minkowski), No. 2).

70 One shall have a look at the extensive presentation in chap. II, III of "corps déformables", in which the equilibrium conditions of such media with euclidean potential are developed using the various possible coordinate systems and according to most manifold specializations.

71 In the Méc. anal. (see 1. Part., sect. IV, § III) Lagrange has particularly emphasized the relevance of this perception also for continua. 
Here, Truesdell writes "only certain” while Hellinger assumes that all mechanics can be framed with Lagrangian methods.

«it presents them systematically and as a branch of mathematics: "Ceux qui aiment l'Analyse, verront avec plaisir la Méchanique en devenir une nouvelle branche,. . . ." The physics and the applications are omitted. He who will apply and interpret the theory, or dwell upon the intricacies and mysteries of its place among the relations between mind and external nature, is expected to learn it first.»

This comment is epistemologically very naive. Archimedean tradition of mechanics is teaching us that the true understanding of physical reality is obtained via the formulation of rigorous mathematical models. It is ironic that Truesdell, i. e. the champion of mathematical formalism, criticized Lagrange because of his rigorous mathematical presentation of mechanics.

«While the knowledge he thus acquires does not of itself put applications into his hands, it gives him the tools to fashion them efficiently, or at least to classify, describe, and teach the applications already known. By consistently leaving applications to the appliers, Lagrange set them on common ground with the theorists who sought to pursue the mathematics further: Both had been trained in the same workshop and spoke the same jargon. Even today this comradeship of infancy lingers on, provided discrete systems and rigid bodies exhaust the universe of mechanical discourse.»

The previous statements are baroque, difficult to follow and fundamentally polemical. The rhetoric trick is very wellknown since Demosthenes: the conclusion is completely unrelated with the previous half-polemical comments. We share with Hellinger the opinion that Lagrangian methods does not apply ONLY to finite dimensional systems.

«In 1788 the mechanics of deformable bodies, which is inherently not only subtler, more beautiful, and grander but also far closer to nature than is the rather arid special case called "analytical mechanics", had been explored only in terms of isolated examples, brilliant but untypical. Unfortunately most of these fitted into Lagrange's scheme; those that did not, he passed over in silence.»

Hellinger's article proves that the last paragraph has a completely false content. Truesdell applies the infamous logical deduction scheme: ex falso (sequitur) quodlibet, EFQ, or translated "from falsehood, anything (follows)", i. e. the principle of Pseudo-Scotus. We share with Hellinger the persuasion that all continuum mechanics falls in the scope of Lagrangian methods. Hellinger includes in Lagrangian (or Variational) Mechanics also dissipative systems by considering the postulation scheme which is usually attributed to Hamilton and Rayleigh. ${ }^{72}$ In this postulation scheme to the Lagrangian action functional one adds a suitable Hamilton-Rayleigh dissipation functional for accounting for dissipation and formulates the principle of virtual work by adding to the first variation of action the variation (with respect to the time derivative of the virtual displacement) of the dissipation functional, with a negative sign and replacing in this last variation the time derivative of the virtual displacement with the virtual displacement (for more details consult e.g. [22] and references cited therein). With the generalization due to Hamilton and Rayleigh it is possible to formulate all equations of mathematical physics which are known in a variational frame (see again [22] or, just to cite a few works $[7,43,50,74]$ ). However, there are results (see e.g. [71]) which seem to indicate that, by suitably embedding it into a larger dimensional system or by introducing Lagrangians suitably depending on time, also a dissipative system can be studied as a (strictly) Lagrangian system, i.e. without introducing Hamilton-Rayleigh dissipation functionals. While such results are considered controversial, we will shortly cite here that sometimes it has been conceived to describe (macroscopically) dissipative systems with conservative models in which many unobservable (microscopic) degrees of freedom trap some of the energy initially present at macro level. This possibility is rigorously included in the Lagrangian postulation by the Liouville Theorem and its consequence the Poincaré Recurrence Theorem (see e.g. [4]): given a Lagrangian system with finite energy, then there exists a recurrence time (eventually very large) after which the system will return back in the neighborhood of any initial configuration. On p. 71 in [4], Arnold suggests that a system of particles trapped in half a box by a diaphragm also after the removal of the diaphragm can be modeled by a Lagrangian even if its behavior is apparently irreversible. The apparent paradox being the fact that the recurrence time is very large (as it has to happen for every apparently macroscopically irreversible systems). In this way Arnold seems to suggest that Lagrangian models DO include all possible models for all physical systems.

Hence, the equilibrium conditions can be classified just as the common problem of the calculus of variations, to determine inside a given domain $V_{0}$ of the variables $a, b, c$ the functions $x, \ldots, \lambda, \ldots$ thereof, such that a certain

72 The Hamilton-Rayleigh principle is not, stricto sensu, a principle of least action. Indeed, it is not formulated in terms of the first variation of a functional. However, as remarked by Hellinger himself, the principle of virtual work is formulated in such a way that it has the structure of a stationarity condition. Actually it states that a differential form is vanishing. Of course not all differential forms are exact forms, and in this sense the principle of virtual work is more general than the principle of stationary action. The Hamilton-Rayleigh principle is building the differential form which has to vanish as the sum of two exact forms. The first exact form appears in the variation of one action functional, the second one is obtained by the variation of a dissipation functional, which then can also be applied to virtual displacements. 
spatial and surface integral including these functions and their derivatives becomes an extremum - for possibly yet undetermined boundary values; differential equations and boundary conditions, which originate herefrom according to the rules of the calculus of variations, correspond exactly to the previously formulated equilibrium conditions.

We cannot delve ourselves into the even more difficult subject of the history of finite elements method, its precursor methods and its relevance in the development of mechanical science. However, we cannot avoid to remark that:

i) In the previous paragraphs it is clearly stated that some boundary conditions are to be assumed "a priori" (these conditions will be called later "essential boundary conditions") before imposing the condition of the minimum of energy while the other boundary conditions (which are necessarily used in the strong formulation of stationarity) are obtained as necessary conditions for extremality of the total energy and are often called "natural boundary conditions". The reader will remark that while formulating numerical integration codes based on stationarity ONLY the essential boundary conditions are imposed, while the natural boundary conditions are automatically computed by the minimizing process.

ii) Hellinger identifies as equilibrium conditions also the boundary conditions calculated when looking for extrema of energy functionals.

iii) In the subsequent paragraphs it is considered the case of variations of kinematical fields which vanish on the boundaries: it is clearly specified that the obtained equilibrium conditions need to be added to some boundary conditions in order to find well-posed problems.

iv) Hellinger shows clearly to be aware of the need of developing a differential calculus in infinite dimensions in order to be able to find maxima and minima of functionals.

Particularly emphasized is often the case, that $\Phi$ is only the potential of the effects applied within the continuum; then to $-\delta \Phi$ in the equilibrium condition a surface integral, the virtual work of the external compressive forces applied to the boundary, is added, and $\delta \Phi$ itself vanishes necessarily only for those virtual displacements, which have the value zero on $S$. For an equilibrium position, thus the potential $\Phi$ of the forces and stresses applied within $V_{0}$ becomes an extremum, compared with all neighboring states of deformations, admissible with respect to possibly occurring constraints, [and] for [those states of deformations in] which every boundary particle of $V_{0}$ is located at the same point as in the equilibrium position; certainly, the solution of this variational problem is only determined when the position of the boundary particles, i.e. the boundary conditions of the deformation functions, are given directly.

The main interest, which is associated with this formulation, belongs to the question, if here, as in the mechanics of discrete masses, depending on the type of extremum of $\Phi$ also the type of equilibrium is determined, in particular, if Dirichlet's stability criterion ${ }^{73}$ holds, that for the appearance of a stable equilibrium the occurrence of an effective minimum is crucial. The general answer of this question can only be founded on the theory of the motion of the continuum, and indeed it depends, if in the case of an effective minimum $\Phi$ a motion out of the equilibrium state caused by small impulses takes place always in the arbitrary neighborhood of exactly this state of deformation. However, in doing that one can interpret the notion of "arbitrary neighborhood" differently, depending on whether one bounds the distance of every individual particle from its equilibrium position, or [one] imposes this requirement only in average for the whole continuum or for individual subdomains; one obtains accordingly various types of stability.

The fact that Hellinger is a first class mathematician appears here clearly. Remark that the translated article was published in 1913, where we are at the very beginning of functional analysis. Gâteaux was very close to die during World War I after having introduced his derivative and Tonelli was starting to develop his direct methods in the calculus of variations. Hellinger describes the methods to be used in order to characterize the stability of configurations for infinite dimensional systems. He is aware of the fact that not all norms are equivalent in infinite dimensional systems: indeed, he warns the reader about the fact that different concepts of "neighborhood" are possible in the considered context. Being a very measured and self-critical scientist, Hellinger avoids to refer to his famous distance (which he introduced in 1909), which could have been cited here. In the following paragraphs one finds a concise and effective account of the state of the art updated to 1913.

73 P. L. Dirichlet, Journ. f. Math. 32 (1846), p. 85 = Werke II (Berlin 1897), p. 5. 
Apart from the cases of the ordinary theory of elasticity, where the circumstances are very easy ${ }^{74}$, only for a few problems complete analyses of stability have been carried out; and moreover, mostly Dirichlet's criterion or an equivalent theorem is taken directly as a basis. ${ }^{75}$ With reference to this circumstance and to the difficulty, which the direct transition of Dirichlet's proof to continua is opposed to, A. Kneser ${ }^{76}$ has shown the validity of Dirichlet's criterion for the catenary; for the problem of the elastic line M. Born ${ }^{77}$ has elaborated the proof with explicit use of Osgood's theorem ${ }^{78}$ of the calculus of variations in a way [which is] also applicable to other onedimensional problems. Nevertheless in general, for multidimensional integrals, Osgood's theorem and therefore also Dirichlet's criterion may not hold without further ado. ${ }^{79}$

\section{7e. Direct determination of the stress components.}

Without telling it explicitly, in this section Hellinger leaves an original contribution to mechanical science, whose importance cannot be overestimated, especially in computational mechanics: note that the formulated variational principle holds in the general nonlinear regime. Due to the presentations of the same variational principle, including also boundary terms, in Reissner [66, 67], this principle is generally referred to as the Hellinger-Reissner principle. On pp. 2.14-2.15 of [40], Reissner translates the present No. 7e into English and gives an astonishing commentary in which he questions the historical relevance of Hellinger's contribution due to technical details:

«While the absence of any consideration of boundary integrals in the above is generally known, other difficulties appear not to have been noted previously. These include the entirely casual reference to the matter of the invertibility of the relations $s_{i j}=\partial \phi / \partial z_{j, i}{ }^{80}$ (which is, of course, a much more significant restriction than the corresponding condition for $\sigma_{i j}=\partial \Sigma / \partial \varepsilon_{i j}$ ), the absence of a concern with conditions on $\phi$ or $H$ so as to ensure moment equilibrium, and, most importantly, the unqualified conclusion concerning the statement of a general variational theorem for stresses alone, as an obvious consequence of $(1.38)^{81}$, with this clearly being the purpose of this section, given the wording of the heading of the section. Altogether, these difficulties make it questionable whether it is in fact historically meaningful to consider Hellinger's considerations as a stepping-stone to the variational theorem for displacements and stresses in Ref. $[15]^{82} . »$

Concretely, Reissner demands priority about this variational principle for himself - rather self-confident. Risking again to be considered as a naive version of the Courageous Philologist, we dare to construct a provocative hypothesis concerning mechanical science after World War II. Scientists in mechanics from the United States, thus scientists from the victorious power, tried to demolish the scientific heritage of Europe by slighting the contents of the earlier contributions or by not even citing the correct references. The rise of the English language as the new lingua franca, as discussed in the first part of this exegetic series [23], played amongst others into the hands of Truesdell and Reissner ${ }^{83}$ to rewrite the recent history of mechanics. Thus, we also claim that the "history of mechanical science is written by the victors". Certainly, this provocative statement should be investigated further and more scientifically.

For some purposes a transformation of the principle of minimum energy is important, which is analogue to the so called canonical transformation of the dynamics of discrete media. ${ }^{84}$ At first it involves - when for the sake of brevity, we refer only to the first case of No. $7 a$ - that one introduces in place of the 9 derivatives $x_{a}, \ldots, z_{c}$ as new unknowns, the 9 corresponding components of stress formulated with respect to the initial parameters

$$
X_{a}=\frac{\partial \varphi}{\partial x_{a}}, \quad X_{b}=\frac{\partial \varphi}{\partial x_{b}}, \ldots, Z_{c}=\frac{\partial \varphi}{\partial z_{c}}
$$

74 Cf. the overview in IV 25, No. 21, Tedone-Timpe.

75 See IV 25, No. 21, p. 211.

76 A. Kneser, Journ. f. Math. 125 (1903), p. 189.

77 M. Born, Untersuch. über die Stabilität der elastischen Linie. Preisschrift, Göttingen 1906, Appendix.

78 W. F. Osgood, Amer. Trans. 2 (1901), p. 273; cf. II A 8 a (H. Hahn and E. Zermelo), Remark 11).

79 According to a private communication of A. Haar. However, Haar has proven, that a similar theorem holds again as soon as sufficiently high derivatives appear in the integrand of the variational problem (cf. the report on a presentation in the math. Ges. Göttingen, Jahresber. d. d. Math.-Ver. 19 (1910), p. 254.)

80 With $\phi$ and $s_{i j}$ the potential $\varphi$ and the stress components $X_{a}, X_{b}, \ldots, Z_{c}$ of Hellinger are meant.

81 This corresponds to Eq. (20) in the next section.

82 This corresponds to reference [66] in the paper at hand.

83 Even though Reissner is of German origin, he got his scientific education in the United States and received his United States citizenship in 1945 at the age of 32 .

84 Cf. IV 12, P. Stäckel as well as for instance the presentation of the Jacobi-Hamilion theory in II A 5, No. 31, E. v. Weber. An extension to several independent variables is given by Born $^{77}$, Appendix. 
— provided that the corresponding Jacobians do not vanish. If one determines then

$$
H=\varphi-\sum_{(x y z ; a b c)} x_{a} X_{a}=H\left(x, \ldots ; X_{a}, \ldots, Z_{c}\right)
$$

as a function of $x, y, z$ and the new quantities $X_{a}, \ldots, Z_{c}$, then one shows easily with the help of known methods form the calculus of variations ${ }^{85}$, that the vanishing $\delta \Phi$ is equivalent to the vanishing of the first variation of the integral

$$
\iiint_{\left(V_{0}\right)}\left(H\left(x, \ldots ; X_{a}, \ldots, Z_{c}\right)+\sum_{(x y z ; a b c)} \frac{\partial x}{\partial a} X_{a}\right) d a d b d c
$$

which contains as unknown functions $x, y, z$ together with their (linearly appearing) derivatives and moreover $X_{a}, \ldots, Z_{c}$ without derivatives. Thereof, the new "canonical" form of the equilibrium conditions follow, [which] hold in the interior:

$$
\begin{gathered}
\frac{\partial X_{a}}{\partial a}+\frac{\partial X_{b}}{\partial b}+\frac{\partial X_{c}}{\partial c}-\frac{\partial H}{\partial x}=0 \quad\left(\begin{array}{c}
X, Y, Z \\
x, y, z
\end{array}\right), \\
\frac{\partial H}{\partial X_{a}}+\frac{\partial x}{\partial a}=0 \quad\left(\begin{array}{c}
X, Y, Z \\
x, y, z
\end{array} ; a, b, c\right) .
\end{gathered}
$$

In the theory of elasticity, the equations (22b) play a crucial role, since they give as solution of (19) an explicit expression of the deformation with respect to the stress components.

The characteristics of this new variational principle, that not only the deformation quantities but rather the stress components do appear, finds expression even more for the special case, that the energy density $\varphi$ is independent of the values of the deformation functions $x, y, z$, [that it] depends therefore only on the shape change (in the broadest sense). Then H contains thus only the stress components, and one can substitute (21) with the following variational problem with constraints, which is analogous to the [principle] known in the theory of frameworks as the principle of L. F. Menabrea and A. Castigliano ${ }^{86}$ : The first variation of the integral

$$
\iiint H\left(X_{a}, X_{b}, \ldots, Z_{c}\right) d a d b d c
$$

shall vanish, where for comparison all systems of functions $X_{a}, \ldots, Z_{c}$ are allowed, which satisfy the 3 equations

$$
\frac{\partial X_{a}}{\partial a}+\frac{\partial X_{b}}{\partial b}+\frac{\partial X_{c}}{\partial c}=0 \quad(X, Y, Z)
$$

If one denotes the Lagrange multipliers associated with these three constraints by $x, y, z$, then herefrom the equations (22b) are obtained after all. By elimination of these Lagrange multipliers from (22b), for the 9 unknown functions just the 6 equations follow:

$$
\frac{\partial}{\partial b}\left(\frac{\partial H}{\partial X_{a}}\right)=\frac{\partial}{\partial a}\left(\frac{\partial H}{\partial X_{b}}\right) \quad(a, b, c ; X, Y, Z)
$$

these are the so called compatibility conditions ${ }^{87}$ of the theory of elasticity, which express, that a system of stresses being compatible with the conditions (23a) can in fact be an equilibrium system in a continuum with energy density $\varphi$ or $H$. - This principle of Castigliano becomes particularly important in such cases, where in the medium only stresses of a certain type can appear; the conditions representing these restrictions can easily be added [to the principle] as constraints. ${ }^{88}$

85 Cf. M. Born, l. c. ${ }^{77}$ p. 91 ff.

86 L. P. Menabrea, Torino Mem. (2) 25 (1871), p. 141 and A. Castigliano, Théorie d'équilibre des systèmes élastiques (Turin 1879); cf. IV 29a, No. $7 \mathrm{ff}$., M. Grüning. Cf. also E. and F. Cosserat, Corps déform., p. $26 \mathrm{ff}$. for the case of the one-dimensional continuum.

87 See IV 24, No. 7a, Tedone; cf. also A. Haar and Th. v. Kármán, Gött. Nachr., math.-phys. Kl. 1909, p. $204 f f$.

88 Haar and Kármán, l. c. ${ }^{87}$, p. 212. 


\section{7f. The appropriate approaches to kinetics.}

This section left us without words. Hellinger gives a complete description of what could be called Analytical Continuum Mechanics, which, whatever is said in the preface of [80] exists, is very effective and full of physical meaning (whatever the meaning of this expression could be). Of course a lot remains to be done: the reader is referred to Germain [29, 30] for a more updated report about the state of the art. However, let us remark that Hellinger includes a surprisingly advanced list of concepts, ideas and results.

In the first place, also for moving media the so far considered effects, in which only tenters as parameter, come into consideration. If one summarizes at first the ansatz of No. 7 a with the expression of Hamilton's principle No. 5, (5), (6), then the theorem being analogous to the formulation of $7 d$ is obtained: For the actual motion of the continuum $V_{0}$ within the time interval $t_{0} \leqq t \leqq t_{1}$ the fourfold integral

$$
\int_{t_{0}}^{t_{1}} d t \iiint_{\left(V_{0}\right)} d V_{0}\left\{\frac{1}{2} \varrho_{0}\left(x^{\prime 2}+y^{\prime 2}+z^{2}\right)-\varphi\right\}
$$

has an extremum with respect to its values for all neighboring motions being admissible with respect to possibly occurring constraints, which, at the time instants $t_{0}$ and $t_{1}$, leave the continuum in the same position.

As one does it in the case of finitely many degrees of freedom, one can immediately extend this ansatz, by giving up the special dependency of the integrand on the [terms with] time derivatives. To readily include also the case of oriented particles, one only needs to make the connection to the formulas (10), (12) of No. 5d and to demand analogously like in the beginning of No. 7a: For every virtual displacement, the virtual work of the moving continuum in the time interval $t_{0}, t_{1}$ shall be equal to the variation of a single expression depending only on the respective motion, which shall specifically be a fourfold integral over a known function of the functions of motion and the temporal and spatial derivatives thereof:

$$
\Phi=\int_{t_{0}}^{t_{1}} d t \iiint_{\left(V_{0}\right)} d V_{0} \varphi\left(a, b, c, t ; x, \ldots, \nu ; x_{a}, \ldots, \nu_{c} ; x^{\prime}, \ldots, \nu^{\prime} ; x_{a}^{\prime}, \ldots, \nu_{c}^{\prime}\right),
$$

and [shall] be called action integral. ${ }^{89}$ In that case, the formulas for the force, stress and torque components remain basically unchanged, only for the components of momentum additional equations do appear

$$
X_{t}=-\frac{\partial \varphi}{\partial x^{\prime}}, L_{t}=-\frac{\partial \varphi}{\partial \lambda^{\prime}} l_{1}-\frac{\partial \varphi}{\partial \mu^{\prime}} l_{2}-\frac{\partial \varphi}{\partial \nu^{\prime}} l_{3} \quad\left(x, y, z ; \begin{array}{c}
L, M, N \\
l, m, n
\end{array}\right) .
$$

Also here, E. and F. Cosserat ${ }^{90}$ have followed the assumption of a "euclidean potential", which does not change for an arbitrary motion of the continuum together with its triads being regarded as rigid; Besides the quantities (16) it will also include the (nonholonomic) velocity coordinates with respect to the movable coordinate system

$$
\mathfrak{x}=\alpha_{1} x^{\prime}+\beta_{1} y^{\prime}+\gamma_{1} z^{\prime}, \mathfrak{p}=\alpha_{3} \alpha_{2}^{\prime}+\beta_{3} \beta_{2}^{\prime}+\gamma_{3} \gamma_{2}^{\prime} \quad\left(\begin{array}{c}
\mathfrak{x}, \mathfrak{y}, \mathfrak{z} \\
\mathfrak{p}, \mathfrak{q}, \mathfrak{r} \\
1,2,3
\end{array}\right),
$$

and according to this, the components entering the equations of motion can be determined immediately analogously to (18).

Also here a canonical transformation, analogous to No. $7 e$, can be carried out; if one transforms merely with respect to the time derivatives, then for $\varphi$ being independent of $t$ it appears

$$
E=\varphi-\sum_{(\mathfrak{x} \mathfrak{y} \mathfrak{z})} \mathfrak{x} \frac{\partial \varphi}{\partial \mathfrak{x}}-\sum_{(\mathfrak{p} \mathfrak{q} \mathfrak{r})} \mathfrak{p} \frac{\partial \varphi}{\partial \mathfrak{p}}
$$

the energy density of the moving system. ${ }^{91}$

89 Cf. E. and F. Cosserat, Corps déform., p. 4.

90 “Corps déformables", p. 156ff.

91 M. Born, l. c. p. 94f.; E. and F. Cosserat, Corps déform., p. 171, 219. 
Besides this far-reaching generalization there is to be pointed out additionally a special kind of emergence of the temporal derivatives of the functions of motion in the effects, which appears in frictional effects and similar ones and for which an ansatz appears being in a way analogous to the potential-based approach. If we restrict us, that the stress dyad contains a part depending on the time derivatives of the 9 deformation quantities $x_{a}^{\prime}, \ldots, z_{c}^{\prime}$, then it is about the specialty, that the stress components are just the derivatives of a known function $F\left(x_{a}^{\prime}, x_{b}^{\prime}, \ldots, z_{c}^{\prime}\right)$ with respect to $x_{a}^{\prime}, \ldots, z_{c}^{\prime}$ :

$$
X_{a}=\frac{\partial F}{\partial x_{a}^{\prime}}, \quad X_{b}=\frac{\partial F}{\partial x_{b}^{\prime}}, \ldots, \quad Z_{c}=\frac{\partial F}{\partial z_{c}^{\prime}} .
$$
time):

Additional to the work done during the actual motion, the stress dyad contributes with (computed per unit of

$$
-\sum_{(x y z ; a b c)} X_{a} \frac{d x_{a}}{d t}=-\sum_{(x y z ; a b c)} \frac{\partial F}{\partial x_{a}^{\prime}} \cdot x_{a}^{\prime}=-D\left(x_{a}^{\prime}, \ldots, z_{c}^{\prime}\right) .
$$

If $D$ is a positive definite function of its 9 arguments, then the stresses $X_{a}, \ldots$ always use work, and indeed [they use work] in an amount measured by the function $D ; D$ is called the dissipation function associated to the stresses. ${ }^{92}$ By the way, merely the case of $F$ being a quadratic function of $x_{a}^{\prime}, \ldots$ is effectively used; then it follows

$$
X_{a}=\frac{1}{2} \frac{\partial D}{\partial x_{a}^{\prime}}, \quad X_{b}=\frac{1}{2} \frac{\partial D}{\partial x_{b}^{\prime}} \ldots, \quad Z_{c}=\frac{1}{2} \frac{\partial D}{\partial z_{c}^{\prime}}
$$

In a quite similar way one can also consider the dependency on higher time derivatives and determine the corresponding dissipation functions; this has been carried out by W. Voigt $t^{93}$ for linear dependency of $X_{a}, \ldots$ on the derivatives.

\section{Annotated translation of No. 8 (pp. 658-662)}

\section{Limit cases of the ordinary three-dimensional continuum.}

Eventually it remains to be discussed how one can gain by certain typical limit processes from the theory of the free three-dimensional continuum the foundations of other classes of continua which [have been obtained] so far without direct connection by a purely formal analogy; thereby it is sufficient to relate everything to the case of an existing potential of the most simple form (No. 7 a, beginning).

Another very interesting subject which should be investigated concerns the history of the parallel development of so called "direct" models as opposed to "deduced" models in mechanics. Complexity can be approached from different points of view: one can formulate a direct model, heuristically taking into account the detail of considered phenomena by increasing the set of introduced kinematical descriptors. Otherwise one can try to deduce, via a reduction process, a macroscopic model starting from a microscopic one where the elementary constituents of the considered system have a "simple" behavior and where the complexity is represented by their array in space and in the interaction laws among elementary constituents. In order to answer to polemical objections by Poisson, Piola uses in his later works (see [18]) such an approach for introducing higher gradient models: in Piola's case, the microscopic and macroscopic models have the same dimension. In the present section Hellinger reports the state of the art in the modeling of lower dimensional continuum models as obtained by reducing three dimensional models via an asymptotic expansion.

8a. Infinitely thin plates and wires. Primarily, it is about the theory of media whose extension in one or two dimensions can be considered as infinitesimal (plates and wires). In reality there exists always a threedimensional extended domain $\mathfrak{V}$, which depends on a parameter $\varepsilon$ measuring those very small extensions; we will express the abstract limit cases of infinitesimal extension, when we consider a whole family of domains $\mathfrak{V}$, which in the limit $\varepsilon=0$ - furthermore we may assume: continuously - approach the surface or line element, which the direct approach (s. No. 2c) is based on.

92 Lord Rayleigh (J. W. Strutt), Lond. Math. Soc. Proc. 4 (1873), p. 357.

93 W. Voigt, Kompendium I, p. 459 ff.; Lehrbuch der Krystallphysik, Leipzig 1910, p. 792 ff. 
We regret not to have read the previous paragraphs by Hellinger at the beginning of our studies in continuum mechanics. The ontological intrinsic three-dimensional nature of deformable bodies is here synthetically described «In reality there exists always a three-dimensional extended domain» and the mathematical nature of the abstraction which leads to lower dimensional continua is clearly stated by means of the introduction of the concept of "families" of models depending on a small parameter $\varepsilon$ and in the calculation of suitable limits when this parameter is vanishing. The presentation by Hellinger avoids the objections, which since at least the works by Sextus Empiricus, are used to deny to "reduced" models the status of effective conceptual tools. In Sextus' "Adversus Mathematicos" (Прòs $\mu \alpha \theta \eta \mu \alpha \tau \iota x o u ́ \varsigma$, Pros mathematikous) which is sometimes translated as "Against Professors", he claims that the abstract concepts as those presented here by Hellinger cannot exist (see Russo [68] for a detailed discussion of the role of Sextus and his doctrine as a cause and a consequence of the decadence of Hellenistic science). In particular, Sextus refuses to consider any abstract geometrical entity as model for a real body having a physical reality.

Due to this perception, the theory of plates and wires can be connected to the theory of three-dimensional continua, and in fact already S. D. Poisson has chosen this way consistently for one case ${ }^{94}$ : One is formulating the characteristic quantities for the domain $\mathfrak{V}$ as a function of $\varepsilon$ and arrives at the laws holding for the limit case by the just mentioned process $\lim \varepsilon=0$ or else by the restriction to the first terms in the series expansion with respect to $\varepsilon$. From an axiomatic point of view, this approach would be the consequence of a general continuity postulate, which can be formulated as follows: In a medium, whose shape or physical property depends on a continuously variable parameter, the equations of state change without exception continuously with this parameter.

The presentation of this approach shall be based on the variational principle ${ }^{95}$.

The criticism implicit in the footnote 95 can be extended to even more modern textbooks! For instance it applies to [45], Sect. II, where the equations of beams and plates, in large deformations, are deduced assuming that the three-dimensional subjacent continuum deforms only in linear elastic regimes.

As a typical example a medium is considered, which in the initial state occupies the domain $-\varepsilon \leqq c \leqq+\varepsilon$ lying over the surface element $S_{0}$ of the a-b-plane; let its potential be:

$$
\Phi=\iint_{\left(S_{0}\right)} d a d b \int_{-\varepsilon}^{+\varepsilon} d c \varphi\left(a, b, c ; x, \ldots ; x_{a}, \ldots\right) .
$$

The functions of equilibrium $x=x(a, b, c), \ldots$, which under certain boundary conditions make $\Phi$ to a minimum, will depend now on $\varepsilon$; let them be expandable in a series expansion of $\varepsilon$ and $c$ :

$$
x=x^{(0)}(a, b)+c x_{c}^{(0)}(a, b)+\varepsilon x^{(1)}(a, b)+\varepsilon c x_{c}^{(1)}(a, b)+\cdots \quad(x, y, z) .
$$

If one introduces these expressions in $\varphi$ and subsequently expands $\varphi$ itself with respect to the powers of $\varepsilon$ and $c$, then the [following] series for $\Phi$ is obtained

$$
\Phi=\varepsilon \iint_{\left(S_{0}\right)} \varphi_{0} d a d b+\varepsilon^{2} \iint_{\left(S_{0}\right)} \varphi_{1} d a d b+\cdots
$$

where

$$
\varphi_{0}=2 \varphi\left(a, b, 0 ; x^{(0)}, \ldots ; \frac{\partial x^{(0)}}{\partial a}, \frac{\partial x^{(0)}}{\partial b}, x_{c}^{(0)}, \ldots\right)
$$

depends merely on the functions $x^{(0)}, \ldots$, their first partial derivatives with respect to $a, b$ and the functions $x_{c}^{(0)}, \ldots$, while in $\varphi_{1}, \ldots$ more and more coefficients can enter, [which] appear in the series expansion (2) as functions of $a, b$. The actual problem is now to calculate the equilibrium position of the "infinitely thin" plate (or rather its midsurface $c=0$ ) determined by the limit function

$$
\lim _{\varepsilon=0} x(a, b, 0)=x^{(0)}(a, b) \quad(x, y, z) .
$$

94 For the treatment of the problem of the elastic plate; Mém. de l'Acad., Paris 8 (1829), p. $523 f f$.

95 Such series expansions and limit processes are since Poisson the more or less declared basis for all theories of plates and wires (s. IV 25, No. 13 ff., Tedone-Timpe); but the overall view is complicated by restricting oneself from the beginning to infinitesimal deformations in small subdomains and only afterwards one changes over to the finite deformation of the whole under citation of auxiliary hypotheses. The text follows the exposition, which C. Carathéodory presented for the elastic line in a "Göttinger Vorlesung" in the winter term 1906/7. 
Besides, also the determination of further terms in the series (2) can be important, for instance the functions $x_{c}^{(0)}(a, b)$, which determine the new position of the initial normals of the plate, i.e. the deflection of the material against the geometric shape of the midsurface. These functions belong in fact to the characteristic quantities of the deformation, just because in reality it is not about a strict one- or two-dimensional medium; in the direct theory they are given by the Cosserat triad.

The presentation by Hellinger is a short but elegant resumé of the most important results in asymptotic analysis as applied to reduced order and reduced dimension mechanical models. Remark the very elegant observation about the interpretation of the gradients in the orthogonal direction as Cosserat triad. In the subsequent paragraphs a careful list of difficulties which may arise in the asymptotic expansions involved is considered. Note that Hellinger is aware of the possibility of meeting singular perturbation problems.

Now, since $\Phi$ with respect to the considered boundary conditions shall become a minimum for every $\varepsilon$, according to (3) primarily $\iint_{S_{0}} \varphi_{0} d a d b$ must become a minimum; but this is directly a condition for those functions $x^{(0)}(a, b), \ldots, x_{c}^{(0)}(a, b), \ldots$, where for comparison all the functions are allowed, which satisfy the boundary conditions for $x^{(0)}, \ldots, x_{c}^{(0)}$ induced by the given boundary conditions together with (2).

Now it is possible, that the functions $x^{(0)}, \ldots$ are hereby not yet completely determined, but that only certain relations between them emerge. If thereupon one restricts oneself to functions, which satisfy these relations, then it follows secondly, that $x^{(0)}(a, b), \ldots$ and the functions still entering $\varphi_{1}$ make the second term in the series $\iint_{\left(S_{0}\right)} \varphi_{1} d a d b$ to a minimum, where the boundary conditions emerge analogously as before; if those relations allow for instance for the elimination of $x_{c}^{(0)}, \ldots$, then this new variational principle can contain higher derivatives of the functions $x^{(0)}, \ldots$. If one possibly continues with this procedure, then one obtains for the functions $x^{(0)}, \ldots$ a series of two-dimensional variational problems, which contain higher derivatives and to which constraints can be added.

Carrying out this ansatz, however, a crucial difficulty arises: for the solution of the three-dimensional problem, hereby the expansibility into a series of the form (2) is assumed, i. e. a certain regular behavior of these solutions as functions of a parameter $\varepsilon$ included in the boundary equation of the continuum is demanded. For problems of this kind, the value $\varepsilon=0$ now does not only need to be not a regular point, but it could even be an essentially singular point ${ }^{96}$; the possibility of an expansion (2) remains therefore a priori quite questionable. Hence, as long as the dependency of the solutions on the parameters in the boundary conditions is not explored in detail, in this way, a completely satisfying theory of plates and wires, which goes beyond the disclosure of the formal connection with the properties of the three-dimensional media, is not obtained, and the direct approaches, which have especially been formulated by $E$. and F. Cosserat (see No. 3e, $7 c$ ) remain the only reference for now.

\section{8b. Media with one kinematic constraint.}

The content of this last section is also based on some lectures held by D. Hilbert in Göttingen and based on some illuminating considerations due to Lagrange himself. To our knowledge the subject treated by Hellinger carefully and with great circumspection is not yet completely understood and clarified. Concerning this point we refer to more modern literature to verify that a general conceptual scheme for studying constrained continua is still to be found. Indeed, it is clearly stated in the fundamental paper by Ball [5] that

«Note, however, that the constraint of inextensibility [..] is not included [in the presented treatment]. It seems possible, therefore, that solutions do not in general exist for boundary-value problems of inextensible elasticity, and that a higher order theory is required to make such constraints well behaved.»

Actually, Hilbert's lectures consisted in some hints for studying incompressible fluids and incompressibility which is the only kind of constraint that is treated in Ball's approach. Therefore it is a fortiori a difficult problem, which yet has found only partial solutions to concern the "reduction" process from three-dimensional to one- or two-dimensional continua when the three-dimensional continuum, we start from, is actually subjected to internal constraints. The method of Lagrange multipliers is introduced and applied in the presented context. Without daring to criticize Hellinger, whose standing seems to us indisputable, we however remark that, to our knowledge, in the presented asymptotic expansions it is sometimes necessary to expand in terms of the parameter $\varepsilon$ also the Lagrange multiplier itself. Unless we are not aware of some technical details or we have misunderstood the content of this section, it seems to us that such an expansion is not assumed by Hellinger, although equation (8a) may induce to believe the contrary. A comment is needed here: until we could use

96 E. and F. Cosserat, Paris C. R. 145 (1907), p. 1139; 146 (1908), p. 169. 
more mature and developed theories to understand Hellinger's treatment it was easy for us to reach his level of mastery of the presented subject. On the contrary, this last section was, at least for us, difficult: most likely because the subjacent theory is either not well-developed yet or not well-understood by us.

In principle, one can make equivalent considerations to derive from the theory of the freely deformable continuum the laws of such media which are subjected to constraints, and for which the direct ansatz in No. $4 c$ has been given. To discuss again only one typical easy case, it is about a medium $\mathfrak{M}$, for which there exists the constraint

$$
\omega\left(x, \ldots ; x_{a}, x_{b}, \ldots\right)=0
$$

between its deformation quantities[;] by the way [the constraint] can also contain a,... explicitly. Now in reality such a medium will never be realized strictly in nature, moreover this here is again only an abstraction from considerations of such media $\mathfrak{M}_{\varepsilon}$, which almost satisfy the relation (4). $\mathfrak{M}_{\varepsilon}$ may be characterized by a potential of the form No. 7, (1) with the energy density $\varphi_{\varepsilon}$, and it shall depend on a parameter $\varepsilon$, such that without exception for every equilibrium position

$$
\left|\omega\left(x, \ldots ; x_{a}, \ldots, z_{c}\right)\right|<\varepsilon
$$

holds. We consider now such media $\mathfrak{M}_{\varepsilon}$ for a family of values of the parameter $\varepsilon$ converging to 0 ; according to the above declared general continuity postulate ( $p .31)$, in the limit $\varepsilon=0$ the laws of the behavior of $\mathfrak{M}$ will follow $^{97}$.

$\varphi_{\varepsilon}$ is characterized as follows: besides the deformation functions and the derivatives thereof it depends also on the expression $\omega$ explicitly:

$$
\varphi_{\varepsilon}=\varphi_{\varepsilon}\left(x, \ldots ; x_{a}, \ldots ; \omega\left(x, \ldots ; x_{a}, \ldots\right)\right)
$$

If one considers $\varphi_{\varepsilon}$ especially as a function of the last argument $\omega$, then with increasing $\omega, \frac{\partial \varphi_{\varepsilon}}{\partial \omega}$ shall increase continuously, for $\omega=0$ [it shall] vanish identically for all other arguments and for every interval [which] does not contain 0 , for $\lim \varepsilon=0$ [it shall] have uniformly the limit $\pm \infty$ (depending on whether $\omega \gtrless 0$ ); furthermore, for the value $\omega=0$, [the function] $\varphi_{\varepsilon}$ shall have uniformly a limit within the domain of variability coming into consideration.

$$
\lim _{\varepsilon=0} \varphi_{\varepsilon}\left(x, \ldots ; x_{a}, \ldots ; 0\right)=\varphi_{0}\left(x, \ldots ; x_{a}, \ldots\right)
$$

An example of such a function would be $\varphi_{\varepsilon}=\varphi_{0}+\frac{\omega^{2}}{2 \varepsilon}$.

The equilibrium deformation of $\mathfrak{M}_{\varepsilon}$, considering the corresponding boundary conditions, is now determined by the variational principle

$$
\delta \iiint_{\left(V_{0}\right)} \varphi_{\varepsilon}\left(x, \ldots ; x_{a}, \ldots ; \omega\left(x, \ldots ; x_{a}, \ldots\right)\right) d a d b d c=0
$$

For the preparation of the limit process a transformation is used [which is] analogous to the canonical transformation of mechanics. ${ }^{85}$ (Note of the translators: As in the original text, the text refers to the previous footnote 85): Using

$$
\frac{\partial \varphi_{\varepsilon}\left(x, \ldots ; x_{a}, \ldots ; \omega\right)}{\partial \omega}=\lambda
$$

$\omega$ is expressed as a function of $\lambda$ as well as of $x, \ldots ; x_{a}, \ldots$ :

$$
\omega=\bar{\omega}_{\varepsilon}\left(x, \ldots ; x_{a}, \ldots ; \lambda\right)
$$

and thereby the expression

$$
\varphi_{\varepsilon}\left(x, \ldots ; x_{a}, \ldots ; \bar{\omega}\right)-\bar{\omega}_{\varepsilon} \cdot \lambda=H_{\varepsilon}\left(x, \ldots ; x_{a}, \ldots ; \lambda\right)
$$

97 Apparently, Lagrange had such a limit process in mind, as he denoted in his analytical mechanics the multiplier associated to $\omega=0$ as "force", which tries to change the function w; one shall compare in particular Sect. II, No. 9, Sect. IV, No. 6, 18, Sect. V, No. 53, Sect. VII, No. 21 of the first part, as well as the notes of J. Bertrand hereto - meanwhile the transition is not carried out in more detail. The presentation of the text is formulated following suggestions, which D. Hilbert has given in a "Göttinger Vorlesung" in the winter term 1906/7 for the treatment of incompressible fluids. 
as a function of $\lambda, x, \ldots, x_{a}, \ldots$ is set up. Then from the well-known methods of the calculus of variations ${ }^{85}$ it follows that (7) is equivalent to the variational principle

$$
\delta \iiint_{\left(V_{0}\right)}\left\{H_{\varepsilon}\left(x, \ldots ; x_{a}, \ldots ; \lambda\right)+\lambda \cdot \omega\left(x, \ldots ; x_{a}, \ldots\right)\right\} d a d b d c=0
$$

for the four unknown functions $x, y, z, \lambda$.

Herein the limit process can easily be carried out; according to the assumptions on $\varphi_{\varepsilon}, \bar{\omega}_{\varepsilon}\left(x, \ldots ; x_{a}, \ldots ; \lambda\right)$ converges with decreasing $\varepsilon$ uniformly to 0 , and since from (9)

$$
\frac{\partial H_{\varepsilon}\left(x, \ldots ; x_{a}, \ldots ; \lambda\right)}{\partial \lambda}=-\bar{\omega}
$$

follows, under consideration of (6), the uniform existence of the limit

$$
\lim _{\varepsilon=0} H_{\varepsilon}\left(x, \ldots ; x_{a}, \ldots ; \lambda\right)=\varphi_{0}\left(x, \ldots ; x_{a}, \ldots\right),
$$

is easily obtained, which is independent of $\lambda$. Hence, one obtains finally as limit case of (10) the variational principle

$$
\delta \iiint_{\left(V_{0}\right)}\left\{\varphi_{0}\left(x, \ldots ; x_{a}, \ldots\right)+\lambda \cdot \omega\left(x, \ldots ; x_{a}, \ldots\right)\right\} d a d b d c=0 ;
$$

herein one can consider $\lambda$ finally as Lagrange multiplier and has therewith in fact provided exactly the ansatz of No. $4 c$ for a medium with energy density $\varphi_{0}$ and constraint (4). Moreover one can gather from this consideration the relevance of the Lagrange multiplier: according to (8), $\lambda$ is related to the connection of the deformation quantities $\omega$ in the same sense as the stress components $X_{a}$ [are related] to the deformation quantities $x_{a}$ (see No. 7, (4)); it is in a way the stress component associated to this connection $\omega$, more precisely: the factor of $\delta \omega$ in the expression of the virtual work for a medium "almost" satisfying the constraint $\omega=0 .{ }^{97}$ Thus, the reactive effects originating from the occurrence of constraints are to be classified as limit cases of the impressed effects thoroughly considered so far. ${ }^{98}$

\section{Some further conclusions}

When reading the pages which we have translated and commented in the present paper, it becomes clear that Hellinger is a follower of the Lagrangian School. History of science is traversed by memberships to schools since the birth of libraries and written texts. Since the very beginning, the relationship between master and pupil has characterized the process of transmission and improvement of knowledge. The school of (astronomical) mechanics, which moved its first steps presumably with the Epicurean School (having Starchy of Argument as one of the main leading figures), was represented by Conn of Samoa, Archimedes of Syracuse, Eratosthenes of Corene and later by Hipparchus of Nicaean and Seleucus of Seleucid These are the few names of Hellenistic scientists which reached us and whose contribution to science is more or less deducible by available sources (see [68]). Except the personal relation between Conn and Archimedes, which is fully documented, there is no evidence (and in some case the absolute prove of the contrary) of any personal relationship between them. However, they all participated to the great adventure of localizing human beings in their place in the universe. Using logical-deductive theories this great group of Hellenistic scientists formulated (most likely basing their mechanical theories on the principle of virtual work) the mechanical science and applied it to many different problems, including the one concerning the motion of the earth in the solar system. Among other phenomena, they could explain the tides and they started (see the catalog of stars established by Hipparchus) experiments which lasted also millennia (in the case of the observation of the apparent motion of stars from the earth this is indeed the required time span). Hellenistic science has shown what is necessary for the human knowledge to proceed:

i) a school master recognized as a prominent personality - as Archimedes was one;

ii) transmission of knowledge in a formal written form with a rigorous process of selection and review of published books and articles - as organized by Eratosthenes, the librarian at the library of Alexandria;

98 Cf. above No. 7e, p. 27 as well as remark ${ }^{84}$. 
iii) common methods and perseverance in the accumulation of experimental data and development of predictive mathematics - as shown by the coherent activity by Hipparchus and Seleucus;

Centuries may be waited before the work of a scientist finds a successor who is capable to continue it: the Dark Ages prevented any development of the theories of Hipparchus whose first follower resulted to be named Copernicus. This scheme has been reproduced also, in a different time scale, by the perfectly known history of the principle of virtual velocities. Including the change of name: now it is called principle of virtual work. Lagrange, following the intuitions by D'Alembert (whose personal influence was very negative, if we want to believe Truesdell ${ }^{99}$ ), started the modern formalization of the principle of virtual work. Piola was one of his first admirers and continuators, explicitly stating that his intention was to continue his school master's work starting from where death had stopped him to complete it. Also, the Cosserat brothers used the Lagrangian formalism for formulating their more advanced theory of continua and Hellinger laid the basis for very effective computing algorithms by generalizing Lagrangian formulation of continuum mechanics. Hellinger was not very familiar with the content of Piola's contributions. He was much more familiar to the contributions by the Cosserat brothers. It is clear that the babel of languages of science caused some problems in the transmission of knowledge and allowed for some losses, rediscoveries and reformulations of the same concepts in different eras and situations. However, a unity of vision and intents can be found: Analytical Continuum Mechanics has been formulated and developed and supplies a powerful tool for technological applications. Hellinger clarifies also the important contribution to this subject by the German literature: his report about Gauss' formulation of the principle of least constraint is remarkable, among the many other contributions of which he gives a synthetic resumé. Reading Hellinger proves also, once more, that the problem of attribution of scientific results is complicated. Tracking how the ideas of Lagrange, Piola and Hellinger are twisted in some literature is not important for the problem of personal attribution of priority (which is very local in space and time and is, at the end, irrelevant). There is (as underlined in [68]) a more important question which concerns the dynamics of advancement of science which requires to be investigated with scientific methods. Referring to the first paper of this series for a discussion about the efforts of Giambattista Vico in formulating such a new science, we want here to state once more: it is not true that science is advancing linearly, that a more modern text is more detailed, advanced and better grounded than older texts. Still in modern times linguistic and cultural barriers are a true problem for the advancement of knowledge. Hellinger's masterpiece clearly shows the genius of Lagrange and of the Lagrangian School, even if Hellinger underestimates the contribution by Piola and even if the discussion by Hellinger of the correct measures of deformation in continuum mechanics is not as complete as in Piola. However, in a later (and more popular) book [79] one can find on pp. 246-247 the following statements:

«While Lagrange's book is a good starting place, experience with it has led me to the following working hypotheses:

1. There was little new in the Méchanique Analitique; its contents derive from earlier papers of LAGRANGE himself ${ }^{10}$ or from works of Euler and other predecessors.

2. General principles or concepts of mechanics are misunderstood or neglected by Lagrange.

3. Lagrange's histories usually give the right references but misrepresent or slight the contents.

When we read Lagrange's sarcastic comment about d'Arcy, “... he even made out of it a kind of metaphysical principle, which he calls the conservation of action..., as if vague and arbitrary names were the essence of the laws of nature and could by some secret virtue raise to final causes some simple consequences of the known laws of mechanics," Hypotheses 3 and 2 suggest that maybe d'Arcy had something. However, this is a bad lead; we find d'Arcy assumes $\dot{\mathbf{H}}=\mathbf{0}$ for bodies that "act on each other in any way, whether by wires, by inflexibles lines, by laws of attraction, etc. ${ }^{11 "}$ As a reason he says only, "It is known that a body, all of whose parts are connected together, cannot take on any motion in virtue of their reciprocal actions."

Turning to Hypothesis 1, we can choose first to follow up Lagrange's own earlier work. Moving slowly backward in his Euvres is a tedious process. The task is lightened by use of a fourth working hypothesis:

4. Lagrange's best ideas in mechanics derive from his earliest period, when he was studying Euler's papers and had not yet fallen under the personal influence of d'Alembert.»

${ }^{10}$ E.g., the famous Lagrangean equations had been derived before, namely in $\$ \$ 7-11$ of his "Théorie de la libration de la lune, et des autres phénomènes qui dependent de la figure non sphérique de cette planète,” Nouv. Mem. Acad. Berlin 1780, 203-309 (1782)= (CEuvres 5, 5-122). Here Lagrange begins by writing the inertial forces in rectangular Cartesian co-ordinates, while the accelerating forces are expressed in terms of distances from "any centers" $(\S 5)$. He states $(\S 13)$ that the Lagrangean equations hold for "an infinity of particles subject to any

99 On p. 248 in [79] on can read that «Lagrange's best ideas in mechanics derive from his earliest period, when he was studying Euler's papers and had not yet fallen under the personal influence of d'Alembert.», so that one must conclude that in Truesdell's opinion Lagrange was a scientist whose weak personality could be easily influenced. 
forces proportional to functions of the distances"; the meaning of this statement is not certain, but with any meaning I can conjecture it is generally false. In this paper Lagrange stops short of deriving the equations of motion of a rigid body by his method.

Likewise, the general integrals derived in the Méchanique Analitique are foreshadowed in the paper, "Remarques générales sur le mouvement de plusieurs corps qui s'attirent mutuellement en raison inverse des carrés des distances," Nouv. Mem. Acad. Berlin 1777, $155-172(1779)=($ Euvres 4, 401-418); this paper rests on the "Newtonian" equations with a "Newtonian" potential function. While in $\S \S$ 4-8 Lagrange derives the integrals of momentum, moment of momentum, and energy, use of special properties of the potential function tends to conceal their meanings. For three bodies, the results are given in $\S \S$ II of his "Essai sur le probleme des trois corps," Prix de l'acad. sci. Paris 9, 1772=Euvres 6, 229-324.

Finally, the principle of virtual work for dynamics, on which the entire Méchanique Analitique is founded, had been given more than twenty years earlier in $\S$ IV of his "Recherches sur la libration de la lune, dans lesquelles on tâche de résoudre la question proposée par l'Académie royale des sciences pour le prix de l'année 1764," Prix de l'acad. sci. Paris 9, 1764 OEuvres 6, 5-61.

${ }^{11}$ See the argument based on Fig. 2 in his "Principe général de dynamique, qui donne la relation entre les espaces parcourus et les temps, quel que soit le système de corps que l'on considère, et quelles que soient leurs actions les unes sur les autres" (read in 1746), Mém. Acad. Sci. Paris 1742, 348-356 (1752), and an addition of 1747, pp. 356-361. The paper is not easy to read. Nothing is added in his later "Réflexions sur le principe de la moindre action de M. de Maupertuis," Mém. Acad. Sci. Paris 1749, 531-538 (1753).

The previous excerpt indicates that Truesdell seems to have made up for his own technical mediocrity by cultivating a sharp and cutting wit, bolstered by his unusually strong command of English, hoping to intimidate detractors into silence by instilling fear of his undeniable literacy. In this way Truesdell managed to shadow and hide the weak points of his vision of mechanics. For sure Truesdell IS NOT a follower of the Lagrangian school. In contrast, Noll was technically much stronger and managed to "set the house in order" (we will deepen this point in the upcoming last part of the exegetic series), by reordering all available results in nonlinear mechanics in such a way that they became accessible to the great majority of mechanical practitioners. However, while giving a precise and rigorous description of the state of the art in mechanical sciences, Noll managed to hide their origins and the principles used for getting them. Eventually, it has to be stated clearly that

i) as recognized also by Landau and Feynman (among many others) all novel theories have been formulated using Lagrangian variational approaches.

ii) no truly new theories were formulated by using the axiomatics which Truesdell attributed to Noll.

iii) the translated text by Hellinger proves that Noll was aware of its content and simply reformulated it, skipping the variational part.

This last point will need to be substantiated as in our opinion it represents a phenomenon of much more general occurrence as it is usually believed.

\section{References}

[1] Published works of Clifford Ambrose Truesdell, III, Arch. Ration. Mech. An. 70(4), 373-393 (1979).

[2] M. Alonso and E. Finn, Physik (Oldenbourg, 2000).

[3] Aristotle, Minor Works: On Colours. On Things Heard. Physiognomics. On Plants. On Marvellous Things Heard. Mechanical Problems. On Indivisible Lines. The Situations and Names of Winds. On Melissus, Xenophanes, Gorgias, (Loeb Classical Library, 1936), 1936, chap. Mechanical Problems, pp. 327-411, translated by W. S. Hett.

[4] V.I. Arnold, Mathematical Methods of Classical Mechanics, 2nd edition, Graduate Texts in Mathematics, Vol. 60 (Springer, 1989).

[5] J. M. Ball, Convexity conditions and existence theorems in nonlinear elasticity, Arch. Ration. Mech. An. 63(4), 337-403 (1976).

[6] A. Battista, C. Cardillo, D. D. Vescovo, N. L. Rizzi, and E. Turco, Frequency shifts induced by large deformations in planar pantographic continua, Nanomechanics Science and Technology: An International Journal 6(2), 161-178 (2015).

[7] V. Berdichevsky, Variational principles of continuum mechanics: I. Fundamentals (Springer Science \& Business Media, 2009).

[8] N. Challamel, A. Kocsis, and C. M. Wang, Higher-order gradient elasticity models applied to geometrically nonlinear discrete systems, Theor. Appl. Mec. 42(4), 223-248 (2015).

[9] B. D. Coleman and W. Noll, Material symmetry and thermostatic inequalities in finite elastic deformations, Arch. Ration. Mech. An. 15(2), 87-111 (1964).

[10] N. M. Cordero, S. Forest, and E. P. Busso, Second strain gradient elasticity of nano-objects, J. Mech. Phys. Solids 97, $92-124$ (2016), SI:Pierre Suquet Symposium.

[11] M. A. Coxhead, A close examination of the pseudo-Aristotelian mechanical problems: The homology between mechanics and poetry as techne, Stud. Hist. Philos. Sci. A 43(2), 300 - 306 (2012), Structures and Strategies in Ancient Greek and Roman Technical Writing.

[12] F. D’Annibale, G. Rosi, and A. Luongo, Linear stability of piezoelectric-controlled discrete mechanical systems under nonconservative positional forces, Meccanica 50(3), 825-839 (2015).

[13] F. D'Annibale, G. Rosi, and A. Luongo, On the failure of the 'similar piezoelectric control' in preventing loss of stability by nonconservative positional forces, Z. Angew. Math. Phys. 66(4), 1949-1968 (2015). 
[14] F. dell'Isola, U. Andreaus, and L. Placidi, At the origins and in the vanguard of peridynamics, non-local and higher-gradient continuum mechanics: An underestimated and still topical contribution of Gabrio Piola, Math. Mech. Solid. 20(8) (2015).

[15] F. dell'Isola, I. Giorgio, M. Pawlikowski, and N.L. Rizzi, Large deformations of planar extensible beams and pantographic lattices: heuristic homogenization, experimental and numerical examples of equilibrium, P. Roy. Soc. Lond. A Mat. 472(2185) (2016).

[16] F. dell'Isola, M. Guarascio, and K. Hutter, A variational approach for the deformation of a saturated porous solid. A secondgradient theory extending Terzaghi's effective stress principle, Arch. Appl. Mech. 70(5), 323-337 (2000).

[17] F. dell'Isola, A. Madeo, and P. Seppecher, Boundary conditions at fluid-permeable interfaces in porous media: A variational approach, Int. J. Solids Struct. 46(17), 3150 - 3164 (2009).

[18] F. dell'Isola, G. Maier, U. Perego, U. Andreaus, R. Esposito, and S. Forest, The complete works of Gabrio Piola: Volume I: Commented English Translation, No. 38 in Advanced Structured Materials (Springer, 2014).

[19] F. dell'Isola and P. Seppecher, "hypertractions and hyperstresses convey the same mechanical information Continuum Mech. Thermodyn. (2010) 22:163-176" by Prof. Podio Guidugli and Prof. Vianello and some related papers on higher gradient theories, Continuum Mech. Therm. 23(5), 473-478 (2010).

[20] F. dell'Isola, U. Andreaus, and L. Placidi, At the origins and in the vanguard of peridynamics, non-local and higher-gradient continuum mechanics: An underestimated and still topical contribution of Gabrio Piola, Math. Mech. Solid. (2014).

[21] F. dell'Isola, A. Della Corte, and I. Giorgio, Higher-gradient continua: The legacy of Piola, Mindlin, Sedov and Toupin and some future research perspectives, Math. Mech. Solid. (2016).

[22] F. dell'Isola and L. Placidi, Variational principles are a powerful tool also for formulating field theories, (Springer Vienna, Vienna, 2012), 2012, pp. 1-15.

[23] S. R. Eugster and F. dell'Isola, Exegesis of the introduction and sect. I from "Fundamentals of the mechanics of continua" by E. Hellinger, Z. Angew. Math. Mech. 97(4), 477-506 (2017).

[24] R. Feynman, The Feynman Lectures on Physics: The New Millennium Edition (Basic Books, 2013).

[25] Forest, S., Mechanics of generalized continua: construction by homogenizaton, J. Phys. IV France 08(PR4), 39-48 (1998).

[26] E. Fried and M.E. Gurtin, Tractions, balances, and boundary conditions for nonsimple materials with application to liquid flow at small-length scales, Arch. Ration. Mech. An. 182(3), 513-554 (2006).

[27] P. Germain, La méthodes des puissances virtuelles en mécanique des milieux continus - 1ère partie, théorie du second gradient, J. Mécanique 12, 235-274 (1973).

[28] P. Germain, The method of virtual power in continuum mechanics. part 2: Microstructure, SIAM J. Appl. Math. 25, 556-575 (1973).

[29] P. Germain, Toward an analytical mechanics of materials, in: Nonlinear Thermodynamical Processes in Continua, edited by M. W. and G. A. MauginNo. 61 (TUB-Dokumentation und Tagungen, Berlin, 1992), pp. 198-212.

[30] P. Germain, Functional concepts in continuum mechanics, Meccanica 33(5), 433-444 (1998).

[31] I. Giorgio, L. Galantucci, A. Della Corte, and D. Del Vescovo, Piezo-electromechanical smart materials with distributed arrays of piezoelectric transducers: current and upcoming applications, Int. J. Appl. Electrom. 47(4), 1051-1084 (2015).

[32] C. Glocker, Set-Valued Force Laws, Dynamics of Non-Smooth Systems, Lecture Notes in Applied Mechanics, Vol. 1 (Springer, 2001).

[33] C. Glocker, The principles of d'Alembert, Jourdain, and Gauss in nonsmooth dynamics part I: Scleronomic multibody systems, Z. Angew. Math. Mech. 78(1), 21-37 (1998).

[34] M.E. Gurtin, Thermodynamics and the possibility of spatial interaction in elastic materials, Arch. Ration. Mech. An. 19(5), 339-352 (1965).

[35] M.E. Gurtin, A gradient theory of single-crystal viscoplasticity that accounts for geometrically necessary dislocations, J. Mech. Phys. Solids 50(1), 5 - 32 (2002).

[36] M. E. Gurtin and A. I. Murdoch, Surface stress in solids, Int. J. Solids Struct. 14(6), 431 - 440 (1978).

[37] D. Halliday and R. Resnick, Fundamentals of physics (Wiley, 1981).

[38] A. Javili, F. Dell'Isola, and P. Steinmann, Geometrically nonlinear higher-gradient elasticity with energetic boundaries, J. Mech. Phys. Solids 61(12), 2381-2401 (2013).

[39] R. Jenkyns and A. Stallings, The Nature of Things, Penguin classics (Penguin Books Limited, 2007).

[40] H. Kardestuncer, D. Norrie, and F. Brezzi, Finite element handbook, McGraw-Hill reference books of interest: Handbooks (McGraw-Hill, 1987).

[41] C. Kittel, W.D. Knight, and M. A. Ruderman, Berkeley Physics Course: Mechanics, Berkeley Physics Course (McGraw-Hill, 1965).

[42] J.L. Lagrange, Mécanique Analytique (Éditions Jacques Gabay, Sceaux, 1788).

[43] C. Lanczos, The variational principles of mechanics (Courier Corporation, 2012).

[44] L. D. Landau and E. M. Lifshitz, Course of theoretical physics, vol. 1-10.

[45] L. D. Landau and E. M. Lifshitz, Theory of Elasticity, Course of Theoretical Physics, Vol. 7 (Butterworth-Heinemann, 1986).

[46] G. Maugin, Continuum Mechanics Through the Eighteenth and Nineteenth Centuries: Historical Perspectives from John Bernoulli (1727) to Ernst Hellinger (1914), Solid Mechanics and Its Applications (Springer International Publishing, 2014).

[47] P. McLaughlin, The question of the authenticity of the mechanical problems, september 2013, http://www.philosophie.unihd.de/md/philsem/personal/mclaughlin_authenticity_2013_2.pdf.

[48] A. Misra and P. Poorsolhjouy, Identification of higher-order elastic constants for grain assemblies based upon granular micromechanics, MEMOCS 3(3), 285-308 (2015). 
[49] A. Misra and P. Poorsolhjouy, Elastic behavior of 2D grain packing modeled as micromorphic media based on granular micromechanics, J. Eng. Mech. 143(1), C4016005-1-13 (2017).

[50] B. L. Moiseiwitsch, Variational principles (Courier Corporation, 2013).

[51] C. H. Müller and A. Timpe, Die Grundgleichungen der mathematischen Elastizitätstheorie, in: Mechanik, , Enzyklopädie der Mathematischen Wissenschaften mit Einschluss ihrer Anwendungen, Vol. IV/4 (Teubner, 1906), pp. 1-54.

[52] W. Noll, A mathematical theory of the mechanical behavior of continuous media, Arch. Ration. Mech. An. 2(1), 197-226 (1958).

[53] W. Noll, A new mathematical theory of simple materials, Arch. Ration. Mech. An. 48(1), 1-50 (1972).

[54] W. Noll, Lectures on the Foundations of Continuum Mechanics and Thermodynamics, (Springer Berlin Heidelberg, Berlin, Heidelberg, 1974), 1974, pp. 293-324.

[55] L. C. Pagnini and G. Piccardo, The three-hinged arch as an example of piezomechanic passive controlled structure, Continuum Mech. Therm. 28(5), 1247-1262 (2016).

[56] G. Piccardo, G. Ranzi, and A. Luongo, A direct approach for the evaluation of the conventional modes within the GBT formulation, Thin. Wall Struct. 74, 133 - 145 (2014).

[57] L. Placidi, A variational approach for a nonlinear 1-dimensional second gradient continuum damage model, Continuum Mech. Therm. 27(4), 623-638 (2015).

[58] L. Placidi, A variational approach for a nonlinear one-dimensional damage-elasto-plastic second-gradient continuum model, Continuum Mech. Therm. 28(1), 119-137 (2016).

[59] L. Placidi, E. Barchiesi, E. Turco, and N. L. Rizzi, A review on 2D models for the description of pantographic fabrics, Z. Angew. Math. Phys. 67(5), 121 (2016).

[60] L. Placidi, I. Giorgio, A. Della Corte, and D. Scerrato, Euromech 563 Cisterna di Latina 17-21 march 2014 Generalized continua and their applications to the design of composites and metamaterials: A review of presentations and discussions, Math. Mech. Solid. pp. 1-14 (2015).

[61] L. Placidi, L. Greco, S. Bucci, E. Turco, and N. L. Rizzi, A second gradient formulation for a 2D fabric sheet with inextensible fibres, Z. Angew. Math. Phys. 67(5), 114 (2016).

[62] P. Podio-Guidugli and M. Vianello, Hypertractions and hyperstresses convey the same mechanical information, Continuum Mech. Therm. 22(3), 163-176 (2010).

[63] E. Ramm, Principles of Least Action and of Least Constraint, (Springer Berlin Heidelberg, Berlin, Heidelberg, 2014), 2014, pp. 23-43.

[64] G. Ranzi and A. Luongo, A new approach for thin-walled member analysis in the framework of GBT, Thin. Wall Struct. 49(11), $1404-1414$ (2011).

[65] H. Reda, Y. Rahali, J. Ganghoffer, and H. Lakiss, Wave propagation in 3D viscoelastic auxetic and textile materials by homogenized continuum micropolar models, Composite Structures 141, 328 - 345 (2016).

[66] E. Reissner, On a variational theorem for finite elastic deformations, J. Math. Phys. 32, 129-135 (1953).

[67] E. Reissner, On a variational theorem in elasticity, J. Math. Phys. 29(1), 90-95 (1950).

[68] L. Russo, The forgotten revolution: How science was born in $300 \mathrm{BC}$ and why it had to be reborn (Springer, New York, 2004).

[69] J. Salençon, Handbook of Continuum Mechanics: General Concepts, Thermoelasticity, Physics and Astronomy Online Library (Springer, 2001).

[70] J. Salençon, Mécanique des milieux continus: Concepts généraux, Mécanique des milieux continus (École Polytechnique, 2005).

[71] W. Sarlet, The helmholtz conditions revisited. a new approach to the inverse problem of lagrangian dynamics, J. Phys. A - Math. Gen. 15(5), 1503 (1982).

[72] D. Scerrato, I. Giorgio, and N. L. Rizzi, Three-dimensional instabilities of pantographic sheets with parabolic lattices: numerical investigations, Z. Angew. Math. Phys. 67(3), 53 (2016).

[73] G. Sciarra, F. dell'Isola, and O. Coussy, Second gradient poromechanics, Int. J. Solids Struct. 44(20), 6607 - 6629 (2007).

[74] R. L. Seliger and G. Whitham, Variational principles in continuum mechanics, in: P. Roy. Soc. Lond. A. Mat., (1968), pp. 1-25.

[75] S. Silling, Reformulation of elasticity theory for discontinuities and long-range forces, J. Mech. Phys. Solids 48(1), $175-209$ (2000).

[76] P. V. Spade and C. Panaccio, William of Ockham, in: The Stanford Encyclopedia of Philosophy, edited by E. N. Zalta, winter 2016 edition, 2016).

[77] D. K. Trinh, R. Janicke, N. Auffray, S. Diebels, and S. Forest, Evaluation of generalized continuum substitution models for heterogeneous materials, Int. J. Multiscale Comput. Eng. 10(6), 527-549 (2012).

[78] C. Truesdell, Die Entwicklung des Drallsatzes, Z. Angew. Math. Mech. 44(4-5), 149-158 (1964).

[79] C. Truesdell, Essays in the History of Mechanics (Springer, New York, 1968).

[80] C. Truesdell, A First Course in Rational Continuum Mechanics (Academic Press, 1977).

[81] C. Truesdell and W. Noll, The non-linear field theories of mechanics, in: The non-linear field theories of mechanics, edited by S. Flügge, Encyclopedia of Physics Vol. III/3 (Springer, 1965).

[82] C. Truesdell and R. Toupin, The classical field theories, in: Principles of Classical Mechanics and Field Theory, edited by S. Flügge, Encyclopedia of Physics Vol. III/1 (Springer, 1960).

[83] E. Turco, K. Barcz, and N. L. Rizzi, Non-standard coupled extensional and bending bias tests for planar pantographic lattices. Part II: comparison with experimental evidence, Z. Angew. Math. Phys. 67(123), 1-16 (2016).

[84] E. Turco, K. Barcz, and N. L. Rizzi, Non-standard coupled extensional and bending bias tests for planar pantographic lattices. part ii: comparison with experimental evidence, Z. Angew. Math. Phys. 67(5), 123 (2016).

[85] E. Turco, F. dell'Isola, N. L. Rizzi, R. Grygoruk, W.H. Müller, and C. Liebold, Fiber rupture in sheared planar pantographic sheets: Numerical and experimental evidence, Mech. Res. Commun. 76, 86 - 90 (2016). 
[86] E. Turco, M. Golaszewski, A. Cazzani, and N. L. Rizzi, Large deformations induced in planar pantographic sheets by loads applied on fibers: Experimental validation of a discrete lagrangian model, Mech. Res. Commun. 76, 51 - 56 (2016).

[87] E. Turco and N.L. Rizzi, Pantographic structures presenting statistically distributed defects: Numerical investigations of the effects on deformation fields, Mech. Res. Commun. 77, 65 - 69 (2016).

[88] D. D. Vescovo and I. Giorgio, Dynamic problems for metamaterials: Review of existing models and ideas for further research, International Journal of Engineering Science 80, 153 - 172 (2014), Special issue on Nonlinear and Nonlocal Problems. In occasion of 70th birthday of Prof. Leonid Zubov.

[89] J. Wallis, Mechanica, Sive, De Motu, Tractatus Geometricus (Pitt, Londini, 1670).

[90] M. White, The continuous and the discrete: Ancient physical theories from a contemporary perspective (1992).

[91] T. N. Winter, The mechanical problems in the corpus of Aristotle, Faculty Publications, Classics and Religious Studies Department (2007), paper 68.

[92] T. Wright, Elements of Mechanics Including Kinematics, Kinetics and Statics: With Applications (D. Van Nostrand Company, 1896).

[93] Y. Yang, W. Y. Ching, and A. Misra, Higher-order continuum theory applied to fracture simulation of nanoscale intergranular glassy film, J. Nanomech. Micromech. 1(2), 60-71 (2011).

[94] Y. Yang and A. Misra, Higher-order stress-strain theory for damage modeling implemented in an element-free Galerkin formulation, Comput. Model Eng. Sci. 64(1), 1-36 (2010). 